\title{
Steps toward determination of the size and structure of the broad-line region in active galactic nuclei. IV - Intensity variations of the optical emission lines of NGC 5548
}

\section{Citation}

Dietrich, M., W. Kollatschny, B. M. Peterson, J. Bechtold, R. Bertram, N. G. Bochkarev, T. A. Boroson, et al. 1993. "Steps Toward Determination of the Size and Structure of the Broad-Line Region in Active Galactic Nuclei. IV - Intensity Variations of the Optical Emission Lines of NGC 5548." The Astrophysical Journal 408 (May): 416. doi:10.1086/172599.

\section{Published Version}

doi:10.1086/172599

\section{Permanent link}

http://nrs.harvard.edu/urn-3:HUL.InstRepos:30212181

\section{Terms of Use}

This article was downloaded from Harvard University's DASH repository, and is made available under the terms and conditions applicable to Other Posted Material, as set forth at http:// nrs.harvard.edu/urn-3:HUL.InstRepos:dash.current.terms-of-use\#LAA

\section{Share Your Story}

The Harvard community has made this article openly available.

Please share how this access benefits you. Submit a story. 


\title{
STEPS TOWARD DETERMINATION OF THE SIZE AND STRUCTURE OF THE BROAD-LINE REGION IN ACTIVE GALACTIC NUCLEI. IV. INTENSITY VARIATIONS OF THE OPTICAL EMISSION LINES OF NGC 5548
}

\author{
M. Dietrich, ${ }^{1}$ W. Kollatschny, ${ }^{1}$ B. M. Peterson, ${ }^{2}$ J. Bechtold, ${ }^{3}$ R. Bertram, ${ }^{2,4}$ N. G. Bochkarev, ${ }^{5}$ \\ T. A. Boroson, ${ }^{6}$ T. E. Carone, ${ }^{3,7}$ M. Elvis, ${ }^{8}$ A. V. Filippenko, ${ }^{9}$ C. M. Gaskell,${ }^{10,11}$ J. P. HuChra, ${ }^{8}$ \\ J. B. Hutchings, ${ }^{12}$ A. P. KoratKar, ${ }^{13}$ K. T. Korista, ${ }^{2,14}$ N. J. Lame, ${ }^{2}$ A. LaOr, ${ }^{15}$ \\ G. M. MacAlpine, ${ }^{10}$ M. A. Malkan, ${ }^{16}$ C. Mendes de Oliveira, ${ }^{12,17}$ H. Netzer, ${ }^{18}$ \\ J. Penfold,${ }^{12,19}$ M. V. Penston, ${ }^{20,21}$ E. Pérez, ${ }^{22}$ R. W. Pogge, ${ }^{2,23}$ \\ M. W. Richmond, ${ }^{9}$ E. I. Rosenblatt, ${ }^{16,24}$ A. I. Shapovalova, ${ }^{25}$ \\ J. C. Shields, ${ }^{2,9}$ H. A. Smith, ${ }^{12,26}$ P. S. Smith, ${ }^{3}$ W.-H. Sun, ${ }^{16,27}$ \\ U. Thiele, ${ }^{28}$ S. Veilleux, ${ }^{29,30}$ R. M. WAGNeR, ${ }^{2,4}$ B. J. Wilkes, ${ }^{8}$ \\ B. J. Wills, ${ }^{23}$ AND D. WILls ${ }^{23}$ \\ Received 1992 August 27; accepted 1992 October 30
}

\begin{abstract}
We present measurements of optical emission-line flux variations based on spectra of the Seyfert galaxy NGC 5548 obtained between 1988 December and 1989 October during the course of a large-scale international monitoring effort. The data presented here supplement previously published measurements of the UV lines and continuum, optical continuum, and broad $\mathrm{H} \beta$ emission line. All of the measured optical emission lines, $\mathrm{H} \alpha, \mathrm{H} \beta, \mathrm{H} \gamma, \mathrm{He}$ I $\lambda 5876$, and $\mathrm{He}$ II $\lambda 4686$, show the same qualitative behavior as the UV and optical continua, but with short time delays, or lags, which are different for the various lines. We apply cross-correlation analysis to measure the lags between the various lines and the continuum. We find similar lags with respect to the UV continuum for $\mathrm{H} \alpha$ and $\mathrm{H} \beta, 17$ and 19 days, respectively. The lag for $\mathrm{H} \gamma$ is shorter (13 days), only somewhat larger than the lag measured for $\operatorname{Ly} \alpha$ (about 10 days). The helium lines respond to continuum variations more rapidly than the hydrogen lines, with lags of about 7 days for He II $\lambda 4686$ and 11 days for He I $\lambda 5876$.

Subject headings: galaxies: active — galaxies: fundamental parameters - galaxies: individual (NGC 5548) galaxies: nuclei - galaxies: Seyfert
\end{abstract}

\section{INTRODUCTION}

The variability of the broad emission lines in Seyfert galaxies provides an important tool for investigation of the size, structure, and dynamics of the broad-line region (BLR) in active

\footnotetext{
${ }^{1}$ Universitäts-Sternwarte Göttingen, Geismarlandstrasse 11, D-3400 Göttingen, Germany.

${ }^{2}$ Department of Astronomy, The Ohio State University, 174 West 18th Avenue, Columbus, $\mathrm{OH} 43210$.

${ }^{3}$ Steward Observatory, University of Arizona, Tucson, AZ 85721.

${ }^{4}$ Postal address: Lowell Observatory, Mars Hill Road, 1400 West, Flagstaff, AZ 86001 .

${ }^{5}$ Sternberg Astronomical Institute, University of Moscow, Universitetskij prosp. 13, Moscow V-234, Russia.

${ }^{6}$ Kitt Peak National Observatory, National Optical Astronomy Observatories, P.O. Box 26732, Tucson, AZ 85726.

${ }^{7}$ Postal address: Space Science Laboratory, University of California, Berkeley, CA 94720.

${ }^{8}$ Harvard-Smithsonian Center for Astrophysics, 60 Garden Street, Cambridge, MA 02138 .

${ }^{9}$ Department of Astronomy, University of California, Berkeley, CA 94720.

${ }^{10}$ Department of Astronomy, University of Michigan, Ann Arbor, MI 48109.

${ }^{11}$ Postal address: Department of Physics and Astronomy, University of Nebraska, Lincoln, NE 68588.

12 Dominion Astrophysical Observatory, 5071 West Saanich Road, Victoria, BC, Canada V8X 4M6.

${ }^{13}$ Space Telescope Science Institute, 3700 San Martin Drive, Baltimore, MD 21218.

${ }^{14}$ Observatories of the Carnegie Institution of Washington, 813 Santa Barbara Street, Pasadena, CA 91101.

15 Institute for Advanced Study, Princeton, NJ 08540.
}

galactic nuclei (AGNs). The emission lines respond to continuum variations with different characteristic time scales and amplitudes.

Emission-line variability has been studied in more than a dozen variable Seyfert galaxies. Progress in emission-line

${ }^{16}$ Department of Astronomy, University of California, Math-Science Building, Los Angeles, CA 90024

17 Department of Astronomy, University of British Columbia, Vancouver, BC, Canada V6T 1W5.

${ }^{18}$ School of Physics and Astronomy and the Wise Observatory, Tel-Aviv University, Ramat Aviv, Tel Aviv 69978, Israel.

${ }^{19}$ Department of Mathematics, Physics, and Engineering, Mount Royal College, Calgary, Alberta, Canada T3E 6K6

${ }^{20}$ Royal Greenwich Observatory, Madingley Road, Cambridge CB3 0EZ, United Kingdom.

21 Deceased, 1990 December 23.

22 Instituto de Astrofísica, E-38200, La Laguna, Tenerife, Spain.

${ }^{23}$ McDonald Observatory and Department of Astronomy, University of Texas, RLM 15.308, Austin, TX 78712.

${ }^{24}$ Postal address: The Aerospace Corporation, P.O. Box 92957, El Segundo, CA 90009.

${ }^{25}$ Special Astrophysical Observatory, Academy of Sciences, Nizhni Arkhys Stavropolsky Kraj, 357140, Russia.

${ }^{26}$ Department of Physics and Astronomy, Michigan State University, East Lansing, MI 48824.

${ }^{27}$ Institute of Physics and Astronomy, National Central University, 32054 Chung-Li, Taiwan.

${ }^{28}$ Max-Planck-Institut für Astronomie, Königstuhl, D-6900 Heidelberg, Germany.

${ }^{29}$ Lick Observatory, University of California, Santa Cruz, CA 95064.

${ }^{30}$ Postal address: Institute for Astronomy, University of Hawaii, 2680 Woodlawn Drive, Honolulu, HI 96822. 
variability studies has been reviewed by Peterson (1988) and Penston (1991). Results published since these reviews include studies of NGC 4151 (Clavel et al. 1990; Maoz et al. 1991), NGC 5548 (Netzer et al. 1990; Peterson et al. 1990; Wamsteker et al. 1990), Akn 120 (Peterson, Korista, \& Wagner 1989; Peterson \& Gaskell 1991), Fairall 9 (Clavel, Wamsteker, \& Glass 1989; Koratkar \& Gaskell 1989), Mkn 279 (Maoz et al. 1990), and NGC 3783 (Winge et al. 1992; Koratkar \& MacAlpine 1992). In most studies undertaken to date, the information obtained has been limited because of inadequate temporal resolution, too many gaps in the time series, or very limited wavelength coverage.

In order to investigate the structure of the BLR by emissionline variability studies, a sustained program of high temporal resolution is required; intervals between observations of a few days or less are necessary to study the response of the most rapidly varying lines. Furthermore, high signal-to-noise ratio spectra are highly desirable since the observed variations are not always of very large amplitude. In an effort to meet the temporal sampling requirements and provide an adequate data base for detailed analysis of temporal variations of many emission lines, a large international collaboration has undertaken a coordinated multiwavelength monitoring campaign on the Seyfert galaxy NGC 5548. During the period 1988 December to 1989 August, UV spectra were obtained once every four days with the $I U E$ satellite (Clavel et al. 1991; hereafter Paper I). A ground-based program of optical spectroscopy and photometry was carried out simultaneously (Peterson et al. 1991, hereafter Paper II), and this effort is continuing; results of a second year of ground-based monitoring have already been published (Peterson et al. 1992, hereafter Paper III).

Measurements of the optical continuum and $\mathrm{H} \beta$ variations observed during the first year of the campaign have been presented in Papers II and III. In this paper, we present the variations of the other optical emission lines, $\mathrm{H} \alpha, \mathrm{H} \gamma, \mathrm{He}$ I $\lambda 5876$, and $\mathrm{He}$ II 24686 , as measured from the same data. We also present an independent assessment of the $\mathrm{H} \beta$ light curve, which we find to be in excellent agreement with the original analysis of Paper II.

\section{OBSERVATIONS AND DATA ANALYSIS}

The first year of the optical observations of NGC 5548 began in 1988 December and was completed in 1989 October. A complete log of the spectroscopic observations including the Julian dates, telescopes used, aperture sizes, position angles, etc., is provided in Papers II and III.

Asis usual in AGN emission-line variability studies, we use the narrow emission lines seen in the spectra as an internal flux standard. It is therefore critical that we understand the role of aperture effects in the calibration process; whereas the continuum and broadlines come from essentially a point source, the narrow-line region is somewhat extended (Wilson et al. 1989). Thus, the ratio of broad-line to narrow-line flux in a given spectrum is a function of both the spectrograph entrance aperture geometry and the seeing. In order to assess the possible importance of aperture effects, for two epochs, on UT 1989 February 17 (JD 2447574) and UT 1989 May 16 (JD 2447663) we obtained several spectra with different slit widths and different position angles using the $2.2 \mathrm{~m}$ (on JD 2447574) and $3.5 \mathrm{~m}$ (on JD 2447663) telescopes at Calar Alto in Spain. These observations were made under photometric conditions with seeing of $1^{\prime \prime}$ (FWHM). The spectra were taken with slit widths of $1^{\prime \prime}, 2^{\prime \prime}, 3^{\prime \prime}$, and $4^{\prime \prime}$, and position angles P.A. $=0^{\circ}$ and
P.A. $=90^{\circ}$ in order to study the effects of the different instrument configurations on the observed line intensities.

In the following, we describe how the various line measurements were made and how corrections for aperture effects were determined.

\subsection{The $\mathrm{H} \beta$ Emission Line}

An $\mathrm{H} \beta$ light curve for the 1988-1989 observing season has already been presented in Paper II. In Paper II, the $\mathrm{H} \beta /$ [O III] $\lambda 5007$ flux ratio was measured for all of the spectra in which these lines appear, and the data were then placed on an absolute flux scale by multiplying this flux ratio by the [O III] $\lambda 5007$ line flux measured under photometric conditions $\left(5.53 \times 10^{-13} \mathrm{ergs} \mathrm{s}^{-1} \mathrm{~cm}^{-2}\right.$, in the rest frame of NGC 5548). Comparison of the $\mathrm{H} \beta$ light curves obtained with different instruments on various telescopes shows that the individual light curves based on different data sets are offset from one another by a multiplicative factor; the single most important factor contributing to these systematic offsets is that different aperture geometries were employed with different spectrographs, and as a result differing amounts of [O III $\lambda 25007$ flux from the extended narrow-line region are detected by different instruments. This effect is corrected for in Papers II and III by applying empirically determined (from nearly simultaneous data) multiplicative scaling factors to the measurements from the various data sets. It is implicitly assumed in this analysis that each of the data sets from different sources is internally highly homogeneous. This is as good assumption for most of the data sets, but for a few of the data sets it is somewhat questionable. In particular, a wide variety of relatively narrow entrance apertures was employed for the Calar Alto observations (data set " $M$ " in Papers II and III), and as a result this data set almost certainly introduces systematic errors into the $\mathrm{H} \beta$ light curve. Part of the motivation for the present study is to determine the magnitude of these effects and to apply a suitable correction to each of the Calar Alto spectra, depending on the aperture employed for each observation.

The multislit spectra obtained at Calar Alto on JD 2447574 and JD 2447663 have been examined to determine aperture corrections for the narrow-slit data, which are those most subject to aperture effects. We scale the observed fluxes in the $[\mathrm{O}$ III $] \lambda \lambda 4959,5007$ emission lines relative to the measurements obtained through a slit width of $2^{\prime \prime}$, since most of the spectra of the Calar Alto subsample were taken with this slit width. The multislit spectra show the expected result that the intensity of the nuclear broad $\mathrm{H} \beta$ component remains constant for the different slit widths and position angles used. However, because the [O III] emission lines originate in a more extended region, the measured flux in the $[\mathrm{O}$ III $] \lambda \lambda 4959,5007$ emission lines is larger when wider entrance slits are used. We find that the [O III] flux measured through a $1^{\prime \prime}$ slit is smaller by a factor of $0.89 \pm 0.08$ relative to that measured through a $2^{\prime \prime}$ slit, and the [O III] flux measured through a 4 " slit is larger than the $2^{\prime \prime}$ value by a factor of $1.10 \pm 0.06$. Figure $1 a$ shows the scaling factors to correct [O III] $\lambda \bar{\lambda} 4959,5007$ line fluxes for the effects of using different slit widths. We note that the intensity of the narrow $\mathrm{H} \beta$ component, which we estimate from a multicomponent fit as described below, shows the same dependence on the slit width as the [O III $] \lambda \lambda 4959,5007$ lines.

We also find that the intensities of the [O III $] \lambda \lambda 4959,5007$ lines and of the narrow $\mathrm{H} \beta$ component are smaller for a position angle of $90^{\circ}$ compared to P.A. $=0^{\circ}$ with a slit width of $2^{\prime \prime}$. The measured flux from the extended narrow emission-line 


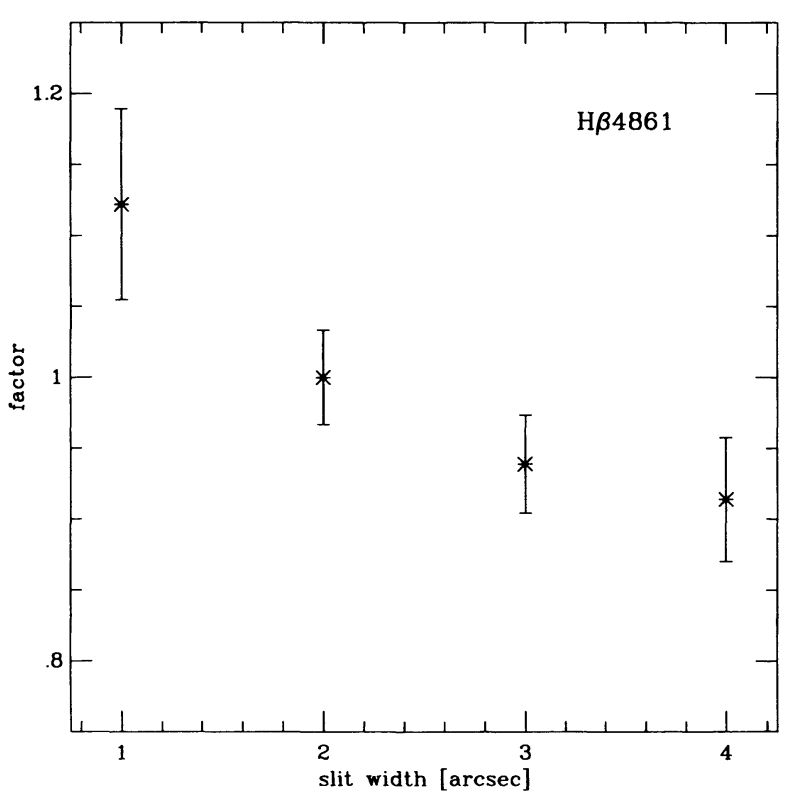

FIG. $1 a$

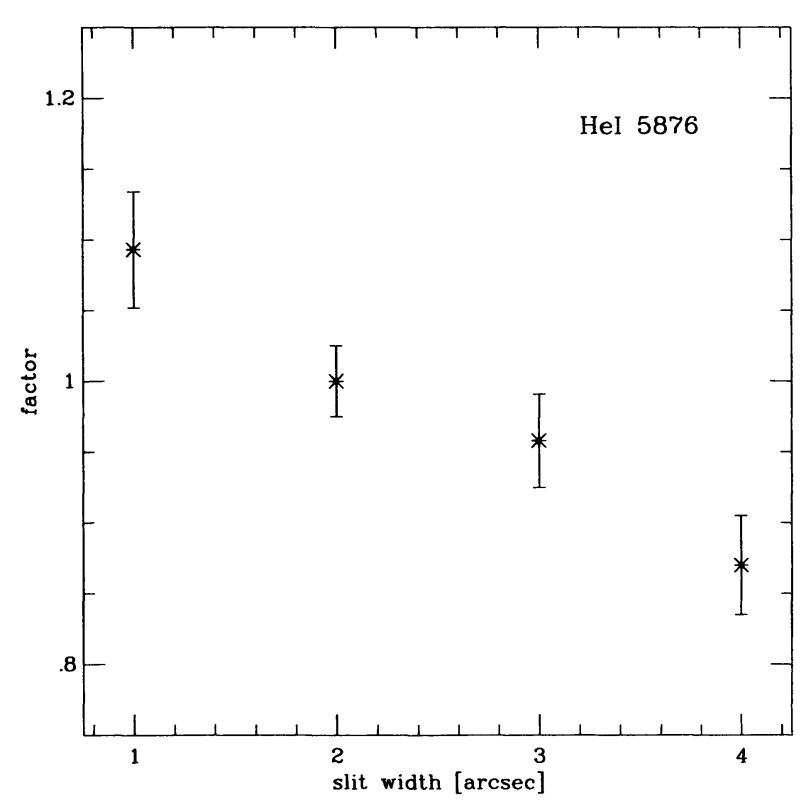

FIG. $1 c$

Fig. 1.-(a) Aperture correction for $\mathrm{H} \beta, \mathrm{H} \gamma$, and $\mathrm{He}$ II $\lambda 4686$, based on the flux in the $[\mathrm{O}$ III] $\lambda \lambda 4959,5007$ narrow lines, is shown as a function of entrance aperture slit width, normalized to the flux obtained through a $2^{\prime \prime}$ slit. This graph provides a correction for properly calibrating, via the narrow-line fluxes, the Calar Alto data obtained through various small apertures. $(b)$ Aperture correction for $\mathrm{H} \alpha$, based on the $[\mathrm{N}$ II] $\lambda \lambda 6548,6584$ and narrow $\mathrm{H} \alpha$ line flux, is shown as a function of slit width, normalized to a 2" slit, as in Fig. 1a. This provides a flux correction for the Calar Alto data obtained through small apertures. (c) Aperture correction for $\mathrm{He}_{\mathrm{I}} \lambda 5876$, based on the $[\mathrm{Fe}$ VII] $\lambda \lambda 5721$ 6087 line fluxes, as a function of slit widths, normalized to the flux through a $2^{\prime \prime}$ slit. This provides a flux correction for the Calar Alto data obtained through small apertures.

region is smaller by $5.2 \pm 1.8 \%$ at $\mathrm{P} . \mathrm{A} .=90^{\circ}$ than at P.A. $=0^{\circ}$. This can be understood by noting the existence of a slightly extended two-sided radio jet structure at P.A. $=160^{\circ}$ (Wilson \& Ulvestad 1982) and the corresponding orientation

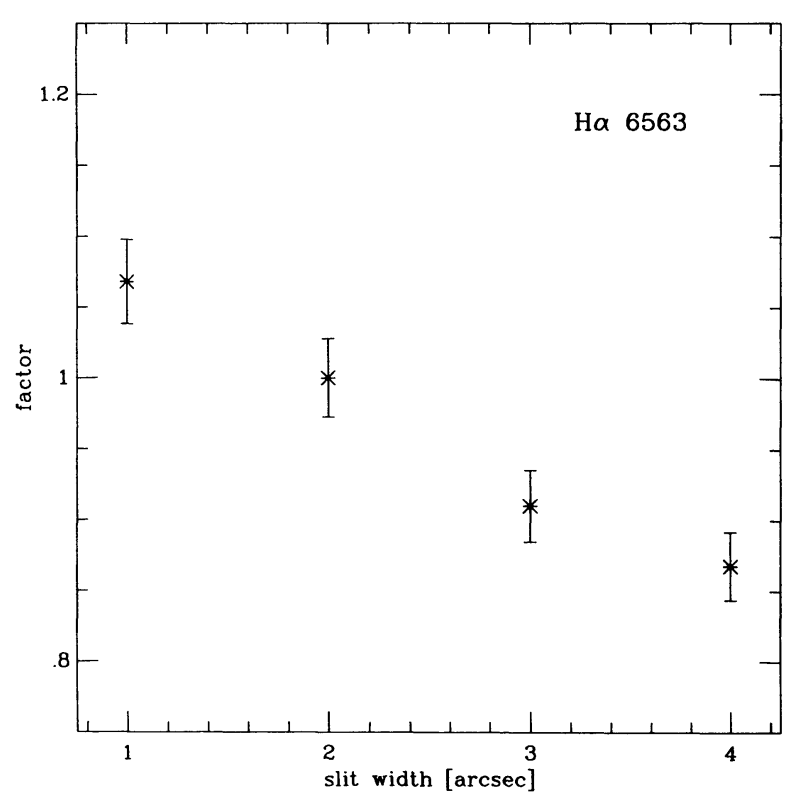

Fig. $1 b$

of the extended [O III $]$ emission at P.A. $=162^{\circ}$ (Wilson et al. 1989).

After determining and applying the correction factors to the Calar Alto measurements only, we proceeded to construct a complete $\mathrm{H} \beta$ light curve in a fashion similar to the process used in Papers II and III. We started from the corrected (to a $2^{\prime \prime}$ slit width and P.A. $\left.=0^{\circ}\right) \mathrm{H} \beta$ measurements from the Calar Alto data set. We note that the effects of seeing variations, as well as possible centering and guiding errors, are not removed by this process, so in fact differences due to these effects appear essentially as random errors. The point-to-point scatter of the corrected fluxes in the $\mathrm{H} \beta$ light curve is found to be quite small, so it therefore can be concluded that the effects of variable seeing and centering and guiding errors must be small.

The $\mathrm{H} \beta$ measurements from the other data sets from the monitoring campaign were then scaled to the $\mathrm{H} \beta$ light curve of the Calar Alto subsample by multiplicative scaling factors which allowed us to correct the light curves from the different data sets for different aperture sizes and position angles. The individual data sets were treated as homogeneous sets and the scaling factors were empirically determined. The final light curve was formed by gradually building up the calibrated data base by including additional data sets, as in Papers II and III. The relative size of scaling factors we used are found to be nearly identical (to within 3\%) to those of Paper II. The different aperture sizes and the corresponding scaling factors are given in Table 1 . The smilarity between the $\mathrm{H} \beta$ light curve obtained here and that given in Paper II clearly demonstrates that the results are robust.

\subsection{The $\mathrm{H} \alpha$ Emission-Line Complex}

$\mathrm{H} \alpha$ spectra were obtained at 99 epochs during the first year of the monitoring campaign. The $\mathrm{H} \alpha$ emission-line complex consists of the narrow $\mathrm{H} \alpha$ and [N II] $\lambda \lambda 6548,6584$ lines, which are assumed to have constant flux, and the broad, variable $\mathrm{H} \alpha$ line. We calibrated the fluxes of the $\mathrm{H} \alpha$ spectra from the sum of the $[\mathrm{N}$ II $] \lambda \lambda 6548,6584$ narrow lines and the narrow $\mathrm{H} \alpha$ component, and effected an absolute flux calibration by measuring 
TABLE 1

SCAle Factors for Data Subsamples

\begin{tabular}{|c|c|c|c|c|}
\hline $\begin{array}{l}\text { Data } \\
\text { Source }\end{array}$ & $\begin{array}{l}\text { Subsample } \\
\text { Code }^{\mathbf{a}}\end{array}$ & P.A. & Aperture Geometry & Scale Factor \\
\hline Ohio State CCD & A & $90^{\circ}$ & $5.0 \times 7.6,1^{\prime \prime} .0 \times 7.6$ & $0.953 \pm 0.020$ \\
\hline $1 \mathrm{~m}$ Wise $\ldots \ldots \ldots \ldots \ldots$ & B & 0 & $20.0 \times 28.0$ & $1.038 \pm 0.000$ \\
\hline INT $2.5 \mathrm{~m} \ldots \ldots \ldots \ldots \ldots$ & $\mathrm{C}$ & $0,72.1,75.4$ & $1.5 \times 6.0$ & $0.930 \pm 0.000$ \\
\hline Hale $5 \mathrm{~m} . . . \ldots \ldots \ldots \ldots$ & $\mathrm{D}$ & $61,66.9$ & $\begin{array}{l}1.0 \times 4.0,1.0 \times 7.0 \\
4.0 \times 8.0\end{array}$ & $0.918 \pm 0.000$ \\
\hline DAO $\ldots$. & $\mathbf{E}$ & 90 & $\begin{array}{l}5.0 \times 8.6, \ldots \\
5.0 \times 18.6\end{array}$ & $0.949 \pm 0.015$ \\
\hline Mt. Hopkins ............. & $\mathrm{F}$ & 90 & $3.2 \times 6.4$ & $0.887 \pm 0.030$ \\
\hline Ohio State IDS ......... & G & & $\varnothing, 7.0$ & $0.953 \pm 0.020$ \\
\hline $3 \mathrm{~m}$ Shane...$\ldots \ldots \ldots \ldots$ & $\mathbf{H}$ & $59 \ldots 130$ & $2.1 \times 7.9$ & $0.903 \pm 0.064$ \\
\hline Steward $\ldots \ldots \ldots \ldots \ldots$ & I & $60,90,130$ & $4.5 \times 27.2$ & $0.924 \pm 0.053$ \\
\hline MDM $\ldots \ldots \ldots \ldots \ldots \ldots$ & $\mathrm{K}$ & 0 & $\begin{array}{l}1.7 \times 3.0,2.4 \times 3.0 \\
6.7 \times 3.0\end{array}$ & $0.949 \pm 0.014$ \\
\hline $6 \mathrm{~m} \mathrm{SAO}$ & $\mathbf{L}$ & & $\varnothing, 2.0$ & $0.943 \pm 0.000$ \\
\hline Calar Alto ............... & $\mathbf{M}$ & 0,90 & $1 \times 10.0 \ldots 4 \times 10.0$ & $1.000 \pm 0.000$ \\
\hline \multirow[t]{2}{*}{$1 \mathrm{~m}$ Nickel .............. } & N1 & 0 & $\begin{array}{l}8.8 \times 9.6,8.8 \times 12.0 \\
8.8 \times 16.0,8.8 \times 16.8\end{array}$ & $1.044 \pm 0.000$ \\
\hline & $\mathrm{N} 2$ & 0 & $4.6 \times 19.2$ & $0.963 \pm 0.000$ \\
\hline $2.1 \mathrm{~m} \mathrm{KPNO} \ldots \ldots \ldots \ldots$ & $\mathrm{O}$ & 90 & $1.9 \times 4.7$ & $0.965 \pm 0.000$ \\
\hline McDonald CCD .......... & $\mathbf{J}, \mathbf{P}$ & 0,90 & $8.0 \times 9.0,7.0 \times 7.2$ & $1.116 \pm 0.000$ \\
\hline McDonald IDS ......... & $Q$ & 90 & $4.4 \times 4.4$ & $0.920 \pm 0.000$ \\
\hline
\end{tabular}

a As in Papers II and III.

the flux in the narrow lines relative to $[\mathrm{O}$ III $] \lambda 5007$. For each spectrum, we determined the intensity of the narrow emissionline fluxes by fitting Gaussian profiles to the observed emission-line profiles. The best fit to the $\mathrm{H} \alpha$ complex was obtained by using five components, as shown in Figure 2, with two separate components to fit the variable broad line, and three components to fit the constant narrow features, $\mathrm{H} \alpha_{\text {narrow }}$ and the $[\mathrm{N}$ II] lines. The profile-fitting program allowed all of the profile parameters (FWHM, position of the peak, and intensity) to vary, but only those solutions which gave narrowline widths and positions consistent with those of the other

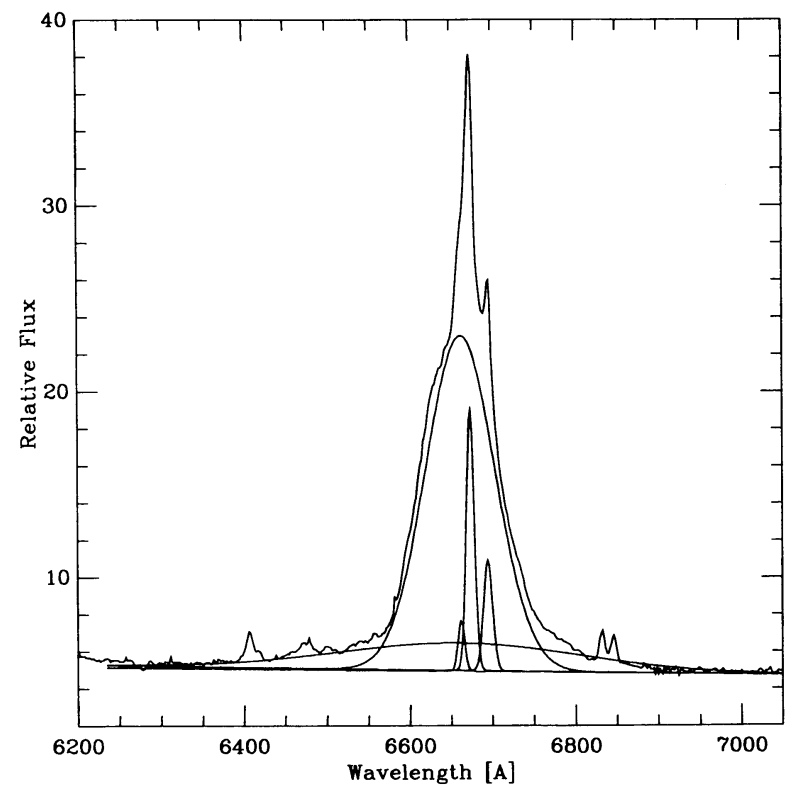

FIG. 2. $-\mathrm{H} \alpha$ line complex is shown in a sample spectrum $(\mathrm{JD}=2447535)$. The complex has been fitted with five Gaussian components as shown in Table 2. narrow emission-lines in the spectrum as well as the theoretical $[\mathrm{N}$ II $]$ line ratio were accepted. The FWHM and relative intensities of these Gaussian components are given in Table 2; note that the uncertainties in the FWHM and the relative flux reflect the rms range of values found in the acceptable fits.

The FWHM of the very broad $\mathrm{H} \alpha$ component $\left(\mathrm{H} \alpha_{v b}\right)$ corresponds to a relative velocity of $13000 \mathrm{~km} \mathrm{~s}^{-1}$. This component is shifted by $900 \mathrm{~km} \mathrm{~s}^{-1}$ to the blue with respect to the narrow lines. This very broad component has been detected previously in the ultraviolet emission lines (Peterson et al. 1990; Wamsteker et al. 1990), and we note that the FWHM and the blueshift of the $\mathrm{H} \alpha_{v b}$ component are comparable to those of the very broad components of the ultraviolet emission lines.

In order to test the efficacy of using the [N II] $\lambda \lambda 6548,6584$ lines and the narrow component of $\mathrm{H} \alpha$ as internal fluxcalibration standards, we divided the intensity of [O III] $\lambda 5007$ by the sum of the intensities of these narrow lines for those spectra which contain both the $\mathrm{H} \alpha$ and $\mathrm{H} \beta$ complexes. We found this ratio to have the constant value $1.91 \pm 0.11$. The small scatter in this ratio demonstrates that our procedure for flux calibration of the $\mathrm{H} \alpha$ data works well.

The flux of the broad $\mathrm{H} \alpha$ line was integrated over the spectral range $6500-6800 \AA$, which corresponds to $6393-6688 \AA$ in the rest frame of NGC 5548 .

Again the Calar Alto multislit spectra were used to determine aperture correction factors for the $\mathrm{H} \alpha$ spectra; the results

TABLE 2

GaUSSIAN COMPONENTS OF H $\alpha$ Fit

\begin{tabular}{ccc}
\hline \hline \multicolumn{1}{c}{ Component } & FWHM $(\AA)$ & Flux $^{\mathrm{a}}$ \\
\hline $\mathrm{H} \alpha_{v b} \ldots \ldots \ldots \ldots \ldots \ldots$ & $290.6 \pm 51.9$ & (variable) \\
$\mathrm{H} \alpha_{\text {broad }} \ldots \ldots \ldots \ldots \ldots$. & $97.3 \pm 3.0$ & (variable) \\
{$[\mathrm{N}$ II $] \lambda 6548 \ldots \ldots \ldots \ldots$} & $11.3 \pm 1.9$ & $0.047 \pm 0.009$ \\
$\mathrm{H} \alpha_{\text {narrow }} \ldots \ldots \ldots \ldots \ldots$ & $13.2 \pm 2.8$ & $0.344 \pm 0.045$ \\
{$[\mathrm{~N}$ II $] \lambda 6584 \ldots \ldots \ldots$} & $12.4 \pm 1.4$ & $0.135 \pm 0.020$ \\
\hline
\end{tabular}

${ }^{\mathrm{a}} F([\mathrm{O} \mathrm{III}] \lambda 5007)=1.00$. 


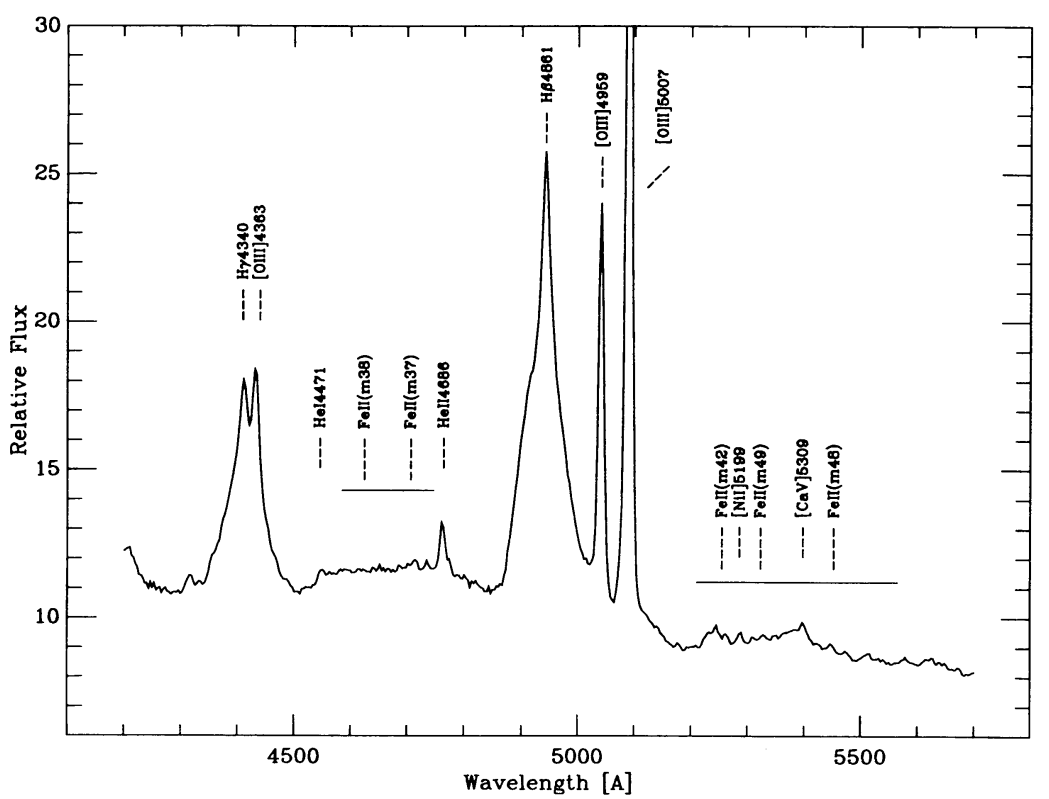

FIG. 3. $-\mathrm{H} \beta$ and $\mathrm{H} \gamma$ line complexes are shown in this sample spectrum $(\mathrm{JD}=2447663)$. The $\mathrm{He}$ II $\lambda 4686$ line which lies between these two features is strongly blended with $\mathrm{Fe}$ II emission.

of this analysis are summarized in Figure $1 b$, which shows the variations of the intensity of the narrow lines in the $\mathrm{H} \alpha$ complex as a function of slit width. To within the uncertainties, the aperture corrections for the $\mathrm{H} \alpha$ complex are identical to those for the $\mathrm{H} \beta$ complex. Similarly, the dependence on the slit position angle is found to be the same for the $\mathrm{H} \alpha$ and $\mathrm{H} \beta$ complexes; the measured intensity of the narrow $\mathrm{H} \alpha$ and [N $\mathrm{NI}]$ lines are smaller by a factor of $1.059 \pm 0.023$ for P.A. $=90^{\circ}$ than for P.A. $=0^{\circ}$.

\subsection{The $\mathrm{H} \gamma$ Emission-Line Complex}

Spectra covering the $\mathrm{H} \gamma$ region (Fig. 3) were obtained at only 58 epochs during the $1988-1989$ observing season as in most cases interest was focused on the stronger $\mathrm{H} \alpha$ and $\mathrm{H} \beta$ complexes.

The $\mathrm{H} \gamma$ emission-line complex is comprised of the broad, variable $\mathrm{H} \gamma$ emission line, plus the constant narrow-line component of $\mathrm{H} \gamma$ and narrow [O III] $\lambda 4363$ emission. The broad $\mathbf{H} \gamma$ feature is also contaminated somewhat by broad, blended Fe II emission lines (see also $\S 2.6$ ). We measured the relative strengths of the components in the $\mathrm{H} \gamma$ line complex in a fashion similar to that which we used to analyze the $\mathrm{H} \alpha$ complex, namely by a multicomponent Gaussian fit. In this case, three components, summarized in Table 3, were used. We note that the very broad component which is detected in $\mathrm{H} \alpha$ and the UV lines cannot be seen in $\mathrm{H} \gamma$ on account of the presence of many contaminating features in this part of the spectrum.

TABLE 3

Gaussian COMPONENTS of $\mathbf{H} \gamma$ Fit

\begin{tabular}{ccc}
\hline \hline Component & FWHM $(\AA)$ & Flux $^{\mathrm{a}}$ \\
\hline $\mathrm{H} \gamma_{\text {broad }} \ldots \ldots \ldots \ldots \ldots$ & $74.6 \pm 9.3$ & (variable) \\
$\mathrm{H} \gamma_{\text {narrow }} \ldots \ldots \ldots \ldots \ldots$ & $14.0 \pm 4.1$ & $0.039 \pm 0.008$ \\
{$[\mathrm{O}$ III $] \lambda 4363 \ldots \ldots \ldots$} & $12.6 \pm 2.6$ & $0.109 \pm 0.012$ \\
\hline${ }^{\mathrm{a}} F([\mathrm{O} \mathrm{III}] \lambda 5007)=1.00$. &
\end{tabular}

The intensity of the broad $\mathrm{H} \gamma$ line was scaled with respect to the intensity of the $[\mathrm{O} \mathrm{III}] \lambda \lambda 4959,5007$ lines. This ratio was corrected for aperture effects in the same way that the $\mathrm{H} \beta$ corrections were made, and the final $\mathrm{H} \gamma$ light curve was derived in the same way as the $\mathrm{H} \beta$ light curve.

\subsection{The $\mathrm{He}$ I $\lambda 5876$ Emission Line}

The spectral range which includes the $\mathrm{He}$ I $\lambda 5876$ line was observed at 82 epochs, sometimes along with the $\mathrm{H} \beta$ spectral region, and sometimes with the $\mathrm{H} \alpha$ complex. The $\mathrm{He}$ I $\lambda 5876$ line flux was obtained by integrating over the spectral range $5850-6125 \AA$, corresponding to $5753-6024 \AA$ in the rest frame of the galaxy. We calibrated these measurements by using the nearby [Fe VII] $\lambda \lambda 5721,6087$ narrow emission lines (Fig. 4). Since these lines show marginal broad components at the bottom of their profiles, only the narrow cores of these lines were measured. The assumption of constant intensity of these narrow-line components was verified by comparing the measured narrow [Fe vII] fluxes with those of the [O $\mathrm{III}] \lambda 5007$ line. We find that the $[\mathrm{O} \mathrm{III}] \lambda 5007 /[\mathrm{Fe}$ VII $] \lambda 687$ flux ratio has the constant value $15.73 \pm 0.57$. We also checked the dependence of the narrow [Fe VII] line intensity on slit width, and find the same dependence as for the [O III] $\lambda \lambda 4959,5007$ and $[\mathrm{N}$ II $] \lambda \lambda 6548,6584$ lines. However, we find no dependence on slit position angle for $[\mathrm{Fe}$ VII $] \lambda 6087$. The correction factors for the $[\mathrm{Fe}$ vII $] \lambda \lambda 5721,6087$ lines, as determined from the Calar Alto multislit data, are shown in Figure 1c.

\subsection{The He II $\lambda 4686$ Emission Line}

The spectral region including the $\mathrm{He}$ II $\lambda 4686$ emission line (Fig. 3) was observed for 57 epochs. The flux in the broad He II $\lambda 4686$ line was obtained by integrating over the wavelength range 4670-4850 $\AA$, corresponding to $4593-4770 \AA$ in the rest frame of the galaxy, above a linear continuum between 4300 and $5200 \AA$. The He II $\lambda 4686$ line is severely blended with multiplet 37 and 38 lines of Fe II, but as the optical Fe II lines do not appear to vary on short time scales $(\$ 2.6)$, only a constant value is added to the integrated $\mathrm{He}$ II $\lambda 4686$ flux we 


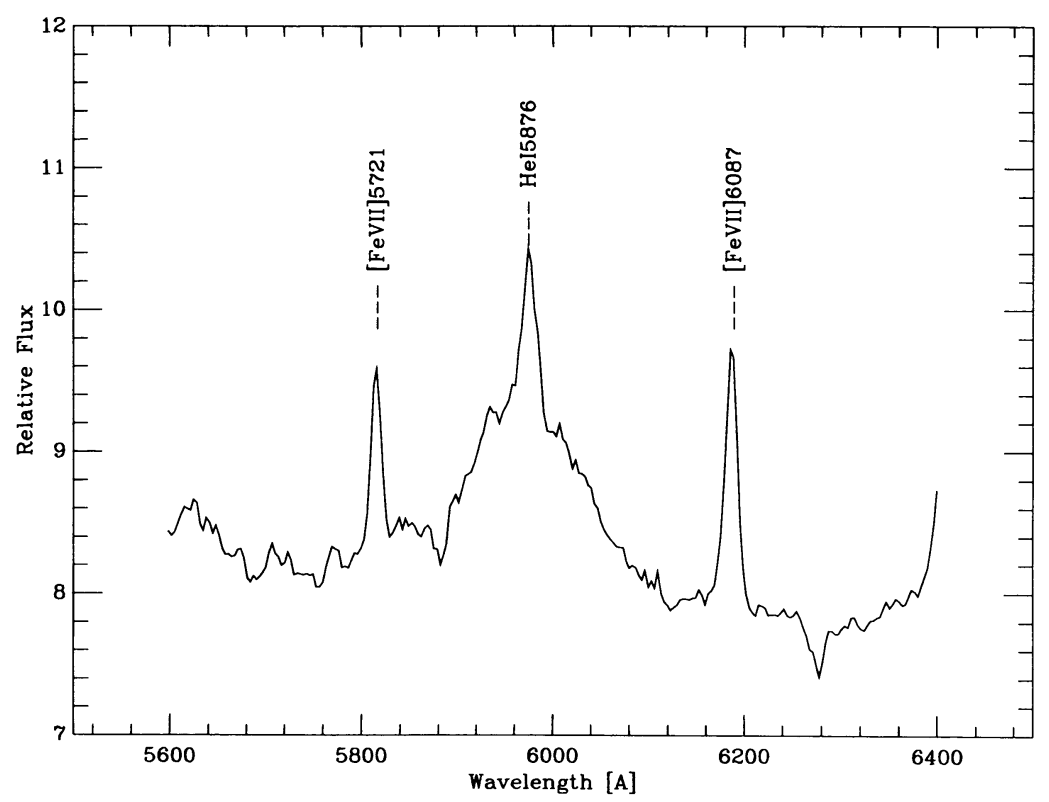

FIG. 4.-Sample spectrum which shows the He I 25876 line $(J D=2447663)$

measure. Flux calibration of the He II $\lambda 4686$ measurements was accomplished in the same fashion as the $\mathrm{H} \beta$ measurements, by reference to the [O III] $\lambda \lambda 4959,5007$ lines, including the appropriate aperture correction as discussed in $\S 2.1$.

\subsection{The Optical Fe II Emission Blends}

The optical spectra of NGC 5548 (Fig. 3) show prominent $\mathrm{Fe}$ II emission comprised of many individual lines primarily from multiplets $37,38,42,48$, and 49 . We measured the flux in the two strongest $\mathrm{Fe}$ II blends over the observed wavelength ranges $4510-4670 \AA$ (multiplets 37 and 38 ) and 5190-5540 $\AA$ (multiplets 48 and 49) for 68 and 97 epochs, respectively. The fluxes of these $\mathrm{Fe}$ II blends were calibrated by reference to the flux in the [O III] $\lambda 4959,5007$ lines, as in the cases of $\mathrm{H} \beta$ and He II 24686 . It is difficult to measure the flux in these blends with high accuracy because they are comparatively broad, lowcontrast features and the measured flux depends critically on how accurately the continuum points on either side of the features can be defined. We find that the mean uncertainty in the measurements, which can be estimated by comparing all pairs of measurements separated in time by 4 days or less, over which time scale little if any real variability is expected, is about $17 \%$. The rms scatter in the light curves (the parameter $F_{\text {var }}$ in the next section) is, however, less than $20 \%$. Thus, any real variations in the optical Fe II blends are lost in the noise. Lack of variability of the optical Fe II emission lines in NGC 5548 was previously noted by Peterson (1987) and Wamsteker et al. (1990) for earlier epochs. We note, however, that during the 1988-1989 observing season, the ultraviolet Fe II features apparently did vary in response to the continuum variations (Maoz et al. 1993).

\section{RESULTS}

\subsection{The Emission-Line Light Curves}

The light curves for the Balmer lines $\mathrm{H} \alpha, \mathrm{H} \beta$, and $\mathrm{H} \gamma$, and for the helium lines He I $\lambda 5876$ and He II $\lambda 4686$, measured as described above, are presented in Table 4. The narrow components of each of these lines, as well as other nonvariable narrow features such as [N II] $\lambda \lambda 6548,6584$, are included in the measurements given in Table 4 since they only add a constant value which does not change the temporal pattern of variability (i.e., the "phase" of the light curves). The light curves of the optical emission lines are shown in Figures $5 a-5 e$. The mean uncertainty for the relative emission-line fluxes is estimated to be 3\% for the stronger emission lines $(\mathrm{H} \alpha$ and $\mathrm{H} \beta), 8 \%$ for the weaker lines ( $\mathrm{He}$ I $\lambda 5876$ and $\mathrm{H} \gamma$ ), and $10 \%$ for the heavily contaminated He II $\lambda 4686$ line.

The pattern of variability of the optical lines is qualitatively the same as seen in the UV lines and UV and optical continua during this period (Papers I-III). Three "events" (outbursts separated by local minima in the light curves) of differing amplitude and duration are clearly seen. The duration of each of the three events is similar for all broad lines, e.g., for $\mathrm{H} \beta, 86$ days for the first event (JD 2447512-JD 2447598), 107 days for the second event (JD 2447598-JD 2447705), and 50 days for the third event (JD 2447705-JD 2447755).

The amplitude of the emission-line variations can be characterized by the parameters $R_{\max }$ and $F_{\text {var }}$, as defined in Paper I. These are, respectively, the ratio of the maximum to minimum flux and the rms of the fluctuations relative to the mean flux, i.e., the relative amplitude of the variations. These parameters are presented in Table 5 for the Balmer and helium lines and the UV and optical continua. We note that inclusion of the constant narrow features in the light curves given here leads to underestimates of both the parameters $R_{\max }$ and $F_{\text {var }}$, although our values are not grossly in error because in each case the constant components comprise a rather small fraction of the total flux in the emission features (cf. Table 6). Both parameters show that the higher order Balmer lines vary with larger amplitude in comparison to the lower order Balmer lines as has been concluded from earlier AGN variability studies (e.g., Antonucci \& Cohen 1983; Wamsteker et al. 1990). He II $\lambda 4686$ varies with a large amplitude, comparable to that of the continuum, and similar to the behavior of He II $\lambda 1640$ (Paper I). 
TABLE 4

EMission-Line Light CURves ${ }^{a}$

\begin{tabular}{|c|c|c|c|c|c|}
\hline $\begin{array}{l}\text { Julian Date } \\
(2440000+)\end{array}$ & $\mathrm{H} \alpha \lambda 6563$ & $\mathrm{H} \beta \lambda 4861$ & $\mathbf{H} \gamma \lambda 4340$ & He I $\lambda 5876$ & He II $\lambda 4686$ \\
\hline 7509. & & $7.40 \pm 0.26$ & $3.55 \pm 0.21$ & $2.74 \pm 0.16$ & $2.51 \pm 0.25$ \\
\hline $7512 \ldots$ & $30.55 \pm 1.06$ & $8.07 \pm 0.28$ & $\ldots$ & $\ldots$ & $\ldots$ \\
\hline $7517 \ldots$ & & $8.09 \pm 0.28$ & $\ldots$ & $\ldots$ & $\ldots$ \\
\hline $7524 \ldots$ & $31.58 \pm 1.11$ & $8.64 \pm 0.30$ & $3.97 \pm 0.24$ & $2.20 \pm 0.13$ & $2.99 \pm 0.30$ \\
\hline $7525 \ldots$ & $31.56 \pm 1.10$ & $8.72 \pm 0.31$ & $3.98 \pm 0.24$ & $2.44 \pm 0.15$ & $2.90 \pm 0.29$ \\
\hline $7528 \ldots$ & $31.25 \pm 1.09$ & $8.82 \pm 0.31$ & $\ldots$ & $2.49 \pm 0.15$ & $\ldots$ \\
\hline $7530 \ldots$ & $32.39 \pm 1.13$ & $8.15 \pm 0.29$ & $4.22 \pm 0.25$ & $\ldots$ & $2.54 \pm 0.25$ \\
\hline 7532 . & $32.24 \pm 1.13$ & $\ldots$ & $\ldots$ & $\ldots$ & $\ldots$ \\
\hline $7533 \ldots$ & $31.16 \pm 1.09$ & $9.22 \pm 0.32$ & $\ldots$ & $2.49 \pm 0.15$ & $\ldots$ \\
\hline $7354 \ldots$ & $\ldots$ & $8.49 \pm 0.30$ & $\ldots$ & $\ldots$ & $\ldots$ \\
\hline $7535 \ldots$ & $32.85 \pm 1.15$ & $8.39 \pm 0.29$ & $\ldots$ & $\ldots$ & $\ldots$ \\
\hline $7539 \ldots \ldots \ldots$ & $32.85 \pm 1.15$ & $9.42 \pm 0.33$ & $4.69 \pm 0.28$ & $2.41 \pm 0.14$ & $3.28 \pm 0.32$ \\
\hline $7543 \ldots$ & $33.54 \pm 1.17$ & $9.04 \pm 0.32$ & $\ldots$ & $2.45 \pm 0.15$ & $\ldots$ \\
\hline $7546 \ldots$ & $34.97 \pm 1.22$ & $9.33 \pm 0.33$ & $4.37 \pm 0.26$ & $2.57 \pm 0.15$ & $3.67 \pm 0.36$ \\
\hline $7549 .$. & $\ldots$ & $9.58 \pm 0.34$ & $4.54 \pm 0.27$ & $\ldots$ & $3.97 \pm 0.40$ \\
\hline $7556 \ldots$ & $\ldots$ & $8.85 \pm 0.31$ & $4.56 \pm 0.27$ & $\ldots$ & $3.14 \pm 0.31$ \\
\hline 7560. & $\ldots$ & $8.98 \pm 0.31$ & $\ldots$ & $\ldots$ & $\ldots$ \\
\hline $7561 \ldots$ & $\ldots$ & $9.18 \pm 0.32$ & $\ldots$ & $\ldots$ & $\ldots$ \\
\hline $7564 \ldots$ & $\ldots$ & $9.12 \pm 0.32$ & $\ldots$ & $\ldots$ & $\ldots$ \\
\hline $7565 \ldots$ & $\ldots$ & $9.15 \pm 0.32$ & $\ldots$ & $\ldots$ & $\ldots$ \\
\hline 7567. & $\ldots$ & $8.74 \pm 0.31$ & $\ldots$ & $\ldots$ & $\ldots$ \\
\hline $7568 \ldots$ & $\ldots$ & $8.70 \pm 0.30$ & $\ldots$ & $\ldots$ & $\cdots$ \\
\hline $7570 .$. & $31.61 \pm 1.11$ & $8.94 \pm 0.31$ & $\ldots$ & $2.32 \pm 0.14$ & $\ldots$ \\
\hline $7571 \ldots$ & $31.51 \pm 1.10$ & $8.65 \pm 0.30$ & $\ldots$ & $2.29 \pm 0.14$ & $\ldots$ \\
\hline 7572. & $31.46 \pm 1.10$ & $8.72 \pm 0.31$ & $\ldots$ & $\ldots$ & $\ldots$ \\
\hline $7573 \ldots$ & $31.55 \pm 1.10$ & $8.77 \pm 0.31$ & $\ldots$ & $\ldots$ & $\ldots$ \\
\hline $7574 \ldots$ & $32.27 \pm 1.13$ & $8.72 \pm 0.31$ & $4.29 \pm 0.26$ & $2.37 \pm 0.14$ & $2.70 \pm 0.27$ \\
\hline $7575 \ldots$ & $31.37 \pm 1.09$ & $8.59 \pm 0.30$ & $\ldots$ & $\ldots$ & $\ldots$ \\
\hline $7576 \ldots$ & $\ldots$ & $8.50 \pm 0.30$ & $4.32 \pm 0.26$ & $\ldots$ & $2.31 \pm 0.23$ \\
\hline 7582. & $\ldots$ & $7.99 \pm 0.28$ & $\ldots$ & $\ldots$ & $\ldots$ \\
\hline $7583 \ldots$ & $30.43 \pm 1.07$ & $8.15 \pm 0.29$ & $3.91 \pm 0.23$ & $2.12 \pm 0.13$ & $2.43 \pm 0.24$ \\
\hline $7584 \ldots$ & $29.18 \pm 1.02$ & $8.46 \pm 0.30$ & $\ldots$ & $\ldots$ & $\ldots$ \\
\hline $7587 \ldots$ & $28.21 \pm 0.99$ & $8.15 \pm 0.29$ & $4.15 \pm 0.25$ & $2.13 \pm 0.13$ & $2.62 \pm 0.26$ \\
\hline $7589 .$. & $29.49 \pm 1.03$ & $7.91 \pm 0.28$ & $3.97 \pm 0.24$ & $1.92 \pm 0.12$ & $2.29 \pm 0.23$ \\
\hline $7590 \ldots$ & $\ldots$ & $7.89 \pm 0.28$ & $4.17 \pm 0.25$ & $\ldots$ & $\ldots$ \\
\hline $7591 \ldots$ & $28.53 \pm 1.00$ & $8.01 \pm 0.28$ & $\ldots$ & $2.13 \pm 0.13$ & $\ldots$ \\
\hline $7592 \ldots$ & $28.72 \pm 1.01$ & $7.87 \pm 0.28$ & $3.80 \pm 0.23$ & $2.15 \pm 0.13$ & $2.52 \pm 0.25$ \\
\hline $7593 .$. & $28.54 \pm 1.00$ & $7.74 \pm 0.27$ & $\ldots$ & $2.13 \pm 0.13$ & $\ldots$ \\
\hline $7594 .$. & $\ldots$ & $7.28 \pm 0.25$ & $\ldots$ & $\ldots$ & $\ldots$ \\
\hline $7597 \ldots$ & $\ldots$ & $7.29 \pm 0.26$ & $\ldots$ & $\ldots$ & $\ldots$ \\
\hline $7598 \ldots \ldots \ldots$ & $30.02 \pm 1.05$ & $7.54 \pm 0.26$ & $\ldots$ & $2.13 \pm 0.13$ & $\ldots$ \\
\hline $7599 \ldots \ldots \ldots$ & $28.35 \pm 0.99$ & $7.36 \pm 0.26$ & $3.79 \pm 0.23$ & $2.21 \pm 0.13$ & $2.80 \pm 0.28$ \\
\hline $7600 \ldots \ldots \ldots$ & $28.11 \pm 0.98$ & $7.32 \pm 0.26$ & $\ldots$ & $2.33 \pm 0.14$ & $\ldots$ \\
\hline $7601 \ldots$ & $\ldots$ & $7.43 \pm 0.26$ & $\ldots$ & $2.23 \pm 0.13$ & $\ldots$ \\
\hline $7606 \ldots \ldots \ldots$ & $\ldots$ & $7.65 \pm 0.26$ & $\ldots$ & $\ldots$ & $\ldots$ \\
\hline $7613 \ldots \ldots \ldots$ & $29.97 \pm 1.05$ & $7.88 \pm 0.28$ & $4.11 \pm 0.25$ & $2.52 \pm 0.15$ & $3.25 \pm 0.32$ \\
\hline $7614 \ldots$ & $31.07 \pm 1.09$ & $8.07 \pm 0.28$ & $4.13 \pm 0.25$ & $2.30 \pm 0.14$ & $2.95 \pm 0.30$ \\
\hline $7615 \ldots$ & $30.71 \pm 1.07$ & $8.27 \pm 0.28$ & $\ldots$ & $2.42 \pm 0.15$ & $\ldots$ \\
\hline $7616 \ldots \ldots \ldots$ & $31.59 \pm 1.11$ & $8.32 \pm 0.29$ & $4.40 \pm 0.26$ & $2.31 \pm 0.14$ & $3.36 \pm 0.33$ \\
\hline $7617 \ldots \ldots \ldots$ & $31.40 \pm 1.10$ & $8.13 \pm 0.28$ & $\ldots$ & $2.30 \pm 0.14$ & $\ldots$ \\
\hline $7618 \ldots \ldots \ldots$ & $31.06 \pm 1.09$ & $8.33 \pm 0.29$ & $\ldots$ & $2.40 \pm 0.14$ & $\ldots$ \\
\hline $7620 \ldots$ & $29.40 \pm 1.03$ & $8.10 \pm 0.28$ & $3.99 \pm 0.24$ & $2.30 \pm 0.14$ & $3.13 \pm 0.31$ \\
\hline $7621 \ldots$ & $31.41 \pm 1.10$ & $8.40 \pm 0.29$ & $4.89 \pm 0.29$ & $2.46 \pm 0.15$ & $2.83 \pm 0.28$ \\
\hline $7622 \ldots$ & $\ldots$ & $8.29 \pm 0.29$ & $4.38 \pm 0.26$ & $2.66 \pm 0.16$ & $3.17 \pm 0.31$ \\
\hline $7623 \ldots$ & $30.91 \pm 1.08$ & $8.55 \pm 0.30$ & $\ldots$ & $2.46 \pm 0.15$ & $\ldots$ \\
\hline $7624 \ldots \ldots \ldots$ & $31.03 \pm 1.09$ & $8.41 \pm 0.29$ & $\ldots$ & $2.47 \pm 0.15$ & $\ldots$ \\
\hline $7626 \ldots$ & $31.46 \pm 1.10$ & $8.42 \pm 0.29$ & $\ldots$ & $2.50 \pm 0.15$ & $\ldots$ \\
\hline $7627 \ldots \ldots \ldots$ & $33.03 \pm 1.16$ & $8.55 \pm 0.30$ & $\cdots$ & $2.61 \pm 0.16$ & $\ldots$ \\
\hline $7628 \ldots \ldots \ldots$ & $\ldots$ & $8.49 \pm 0.30$ & $4.76 \pm 0.29$ & $\ldots$ & $3.61 \pm 0.36$ \\
\hline $7629 \ldots \ldots \ldots$ & $\ldots$ & $8.47 \pm 0.30$ & $\ldots$ & $\ldots$ & $\ldots$ \\
\hline $7631 \ldots \ldots \ldots$ & $32.64 \pm 1.14$ & $8.97 \pm 0.31$ & $4.86 \pm 0.29$ & $2.60 \pm 0.16$ & $3.67 \pm 0.36$ \\
\hline $7632 \ldots \ldots \ldots$ & $\ldots$ & $9.01 \pm 0.32$ & $\ldots$ & $2.75 \pm 0.16$ & $\ldots$ \\
\hline $7642 \ldots \ldots \ldots$ & $\ldots$ & $9.31 \pm 0.33$ & $\ldots$ & $\ldots$ & $\ldots$ \\
\hline $7643 \ldots \ldots \ldots$ & $33.63 \pm 1.18$ & $9.28 \pm 0.32$ & $4.67 \pm 0.28$ & $2.62 \pm 0.16$ & $3.76 \pm 0.37$ \\
\hline $7644 \ldots \ldots \ldots$ & $33.53 \pm 1.17$ & $9.36 \pm 0.33$ & $\ldots$ & $2.66 \pm 0.16$ & $\ldots$ \\
\hline $7645 \ldots$ & $32.65 \pm 1.14$ & $9.38 \pm 0.33$ & $4.97 \pm 0.30$ & $2.70 \pm 0.16$ & $3.75 \pm 0.37$ \\
\hline $7648 \ldots \ldots \ldots$ & $33.04 \pm 1.16$ & $9.45 \pm 0.33$ & $\ldots$ & $\ldots$ & $\ldots$ \\
\hline $7649 \ldots \ldots \ldots$ & $\ldots$ & $9.49 \pm 0.33$ & .. & $2.94 \pm 0.18$ & $\ldots$ \\
\hline $7650 \ldots \ldots \ldots$ & $35.37 \pm 1.24$ & $9.78 \pm 0.34$ & $\ldots$ & $2.75 \pm 0.16$ & $\ldots$ \\
\hline $7652 \ldots \ldots \ldots$ & $35.17 \pm 1.23$ & $9.73 \pm 0.34$ & $\ldots$ & $\cdots$ & $\cdots$ \\
\hline $7653 \ldots \ldots \ldots$ & $\ldots$ & $9.63 \pm 0.34$ & $\cdots$ & $\cdots$ & $\cdots$ \\
\hline
\end{tabular}


TABLE 4-Continued

\begin{tabular}{|c|c|c|c|c|c|}
\hline $\begin{array}{l}\text { Julian Date } \\
(2440000+)\end{array}$ & $\mathrm{H} \propto \lambda 6563$ & $\mathrm{H} \beta \lambda 4861$ & $\mathbf{H} \gamma \lambda 4340$ & He I $\lambda 5876$ & He II $\lambda 4686$ \\
\hline 7654. & $35.12 \pm 1.23$ & $9.76 \pm 0.34$ & & $2.66 \pm 0.16$ & \\
\hline 7655. & & $10.04 \pm 0.35$ & $4.97 \pm 0.30$ & $2.83 \pm 0.17$ & $3.78 \pm 0.37$ \\
\hline $7656 \ldots$ & $33.85 \pm 1.18$ & $9.93 \pm 0.35$ & $\ldots$ & $2.70 \pm 0.16$ & $\ldots$ \\
\hline 7657. & $\ldots$ & $10.00 \pm 0.35$ & $\ldots$ & $\ldots$ & $\ldots$ \\
\hline $7658 \ldots$ & $\ldots$ & $9.48 \pm 0.33$ & $4.86 \pm 0.29$ & $\ldots$ & $3.48 \pm 0.34$ \\
\hline $7660 \ldots$ & $34.58 \pm 1.21$ & $9.61 \pm 0.34$ & $4.89 \pm 0.29$ & $2.87 \pm 0.17$ & $3.62 \pm 0.36$ \\
\hline $7661 \ldots$ & $35.10 \pm 1.23$ & $9.99 \pm 0.35$ & $\ldots$ & $\ldots$ & $\ldots$ \\
\hline $7663 .$. & $35.45 \pm 1.24$ & $10.16 \pm 0.36$ & $4.93 \pm 0.30$ & $2.78 \pm 0.17$ & $3.49 \pm 0.34$ \\
\hline $7664 \ldots$ & $34.31 \pm 1.20$ & $9.76 \pm 0.34$ & $4.94 \pm 0.30$ & $2.65 \pm 0.16$ & $3.27 \pm 0.32$ \\
\hline $7665 \ldots$ & $34.22 \pm 1.20$ & $9.97 \pm 0.35$ & $4.93 \pm 0.30$ & $2.75 \pm 0.17$ & $3.74 \pm 0.37$ \\
\hline 7666. & $34.02 \pm 1.19$ & $9.57 \pm 0.33$ & $\ldots$ & $\ldots$ & $\ldots$ \\
\hline $7668 \ldots$ & $35.95 \pm 1.24$ & $9.60 \pm 0.34$ & $\ldots$ & $\ldots$ & $\ldots$ \\
\hline $7673 \ldots$ & $33.64 \pm 1.18$ & $9.52 \pm 0.33$ & $\ldots$ & $2.62 \pm 0.16$ & $\ldots$ \\
\hline $7674 \ldots$ & $34.11 \pm 1.19$ & $9.54 \pm 0.33$ & $\ldots$ & $2.47 \pm 0.15$ & $\ldots$ \\
\hline 7675. & $34.48 \pm 1.21$ & $9.49 \pm 0.33$ & $\ldots$ & .. & .. \\
\hline $7676 .$. & $34.17 \pm 1.20$ & $9.41 \pm 0.33$ & $\ldots$ & $\ldots$ & $\ldots$ \\
\hline $7678 \ldots$ & $32.07 \pm 1.12$ & $9.27 \pm 0.32$ & $4.46 \pm 0.27$ & $2.52 \pm 0.15$ & $3.53 \pm 0.35$ \\
\hline $7679 .$. & $32.03 \pm 1.12$ & $8.92 \pm 0.31$ & $4.45 \pm 0.27$ & $2.46 \pm 0.15$ & $3.26 \pm 0.32$ \\
\hline $7680 \ldots$ & $31.89 \pm 1.12$ & $9.15 \pm 0.32$ & $4.04 \pm 0.24$ & $2.38 \pm 0.14$ & $3.03 \pm 0.30$ \\
\hline $7681 \ldots$ & $34.77 \pm 1.22$ & $9.41 \pm 0.33$ & $\ldots$ & $2.47 \pm 0.15$ & $\ldots$ \\
\hline $7682 \ldots$ & $34.09 \pm 1.19$ & $9.27 \pm 0.32$ & $\ldots$ & $2.37 \pm 0.14$ & $\ldots$ \\
\hline $7683 \ldots$ & $32.34 \pm 1.13$ & $9.24 \pm 0.32$ & $\ldots$ & $2.27 \pm 0.14$ & $\ldots$ \\
\hline $7684 \ldots$ & $33.82 \pm 1.18$ & $9.08 \pm 0.32$ & $\ldots$ & $2.33 \pm 0.14$ & $\ldots$ \\
\hline $7685 \ldots$ & $34.00 \pm 1.19$ & $8.98 \pm 0.31$ & $\ldots$ & $2.43 \pm 0.15$ & $\ldots$ \\
\hline $7686 \ldots$ & $32.05 \pm 1.12$ & $9.20 \pm 0.32$ & $4.16 \pm 0.25$ & $2.36 \pm 0.14$ & $\ldots$ \\
\hline $7687 \ldots$ & $33.60 \pm 1.18$ & $9.23 \pm 0.32$ & $4.44 \pm 0.27$ & $2.40 \pm 0.14$ & $\ldots$ \\
\hline $7691 \ldots$ & $31.09 \pm 1.09$ & $\ldots$ & $4.14 \pm 0.25$ & $\ldots$ & $\ldots$ \\
\hline $7697 \ldots$ & $29.73 \pm 1.04$ & $8.98 \pm 0.31$ & $\ldots$ & $\ldots$ & $\ldots$ \\
\hline $7699 \ldots$ & $30.32 \pm 1.06$ & $8.77 \pm 0.31$ & $\ldots$ & $\ldots$ & $\ldots$ \\
\hline $7700 \ldots$ & $29.91 \pm 1.05$ & $8.78 \pm 0.31$ & $3.76 \pm 0.23$ & $\ldots$ & $2.97 \pm 0.29$ \\
\hline $7701 \ldots$ & & $8.74 \pm 0.31$ & $3.95 \pm 0.24$ & $\ldots$ & $3.10 \pm 0.31$ \\
\hline $7702 \ldots$ & $30.90 \pm 1.08$ & $8.85 \pm 0.31$ & $4.25 \pm 0.26$ & $2.40 \pm 0.14$ & $3.06 \pm 0.30$ \\
\hline $7703 \ldots$ & $30.75 \pm 1.08$ & $8.64 \pm 0.30$ & $3.87 \pm 0.23$ & $2.25 \pm 0.13$ & $2.75 \pm 0.27$ \\
\hline $7704 \ldots$ & $\ldots$ & $8.14 \pm 0.28$ & $3.90 \pm 0.23$ & $\ldots$ & $2.80 \pm 0.28$ \\
\hline $7705 \ldots \ldots \ldots$ & $30.64 \pm 1.07$ & $8.31 \pm 0.29$ & $\ldots$ & $2.41 \pm 0.14$ & $\ldots$ \\
\hline $7706 \ldots$ & $31.05 \pm 1.09$ & $\ldots$ & $\ldots$ & $\ldots$ & $\ldots$ \\
\hline $7707 \ldots$ & $29.72 \pm 1.04$ & $8.43 \pm 0.30$ & $\ldots$ & $2.45 \pm 0.15$ & $\ldots$ \\
\hline $7708 \ldots \ldots \ldots$ & $31.04 \pm 1.09$ & $8.48 \pm 0.30$ & $\ldots$ & $2.40 \pm 0.14$ & $\ldots$ \\
\hline $7709 \ldots \ldots \ldots$ & $30.39 \pm 1.06$ & $8.67 \pm 0.30$ & $\ldots$ & $2.34 \pm 0.14$ & $\ldots$ \\
\hline $7710 \ldots \ldots \ldots$ & $30.90 \pm 1.08$ & $8.33 \pm 0.29$ & $\ldots$ & $2.37 \pm 0.14$ & $\ldots$ \\
\hline $7711 \ldots \ldots \ldots$ & $\ldots$ & $8.42 \pm 0.29$ & $4.30 \pm 0.26$ & $\ldots$ & $3.40 \pm 0.34$ \\
\hline $7713 \ldots \ldots \ldots$ & $31.41 \pm 1.10$ & $7.90 \pm 0.28$ & $\ldots$ & $\ldots$ & $\ldots$ \\
\hline $7715 \ldots \ldots \ldots$ & $30.81 \pm 1.08$ & $8.10 \pm 0.28$ & $\ldots$ & $2.33 \pm 0.14$ & $\ldots$ \\
\hline $7716 \ldots \ldots \ldots$ & $31.86 \pm 1.12$ & $8.14 \pm 0.28$ & $4.00 \pm 0.24$ & $\ldots$ & $3.24 \pm 0.32$ \\
\hline $7719 \ldots \ldots \ldots$ & $33.84 \pm 1.18$ & $8.74 \pm 0.31$ & $4.13 \pm 0.25$ & $2.30 \pm 0.14$ & $3.39 \pm 0.33$ \\
\hline $7725 \ldots \ldots \ldots$ & $\ldots$ & $9.06 \pm 0.32$ & $4.33 \pm 0.26$ & $\ldots$ & $3.24 \pm 0.32$ \\
\hline $7728 \ldots \ldots \ldots$ & $30.43 \pm 1.06$ & & $\ldots$ & $\ldots$ & $\ldots$ \\
\hline $7730 \ldots \ldots \ldots$ & $28.83 \pm 1.01$ & $8.83 \pm 0.31$ & $\ldots$ & $\ldots$ & $\ldots$ \\
\hline $7736 \ldots \ldots \ldots$ & $\ldots$ & $8.43 \pm 0.29$ & $3.91 \pm 0.23$ & $\ldots$ & $\ldots$ \\
\hline $7741 \ldots \ldots \ldots$ & $\ldots$ & $8.12 \pm 0.28$ & $3.92 \pm 0.24$ & $\ldots$ & $2.46 \pm 0.24$ \\
\hline $7742 \ldots \ldots \ldots$ & $30.31 \pm 1.06$ & $8.07 \pm 0.28$ & $3.50 \pm 0.21$ & $2.04 \pm 0.12$ & $1.95 \pm 0.20$ \\
\hline $7746 \ldots \ldots \ldots$ & $29.61 \pm 1.04$ & $7.48 \pm 0.26$ & $3.07 \pm 0.18$ & $2.16 \pm 0.13$ & $1.90 \pm 0.19$ \\
\hline $7748 \ldots$ & $29.70 \pm 1.04$ & $8.31 \pm 0.29$ & $3.76 \pm 0.23$ & $2.24 \pm 0.13$ & $2.34 \pm 0.23$ \\
\hline $7749 \ldots \ldots \ldots$ & $29.00 \pm 1.01$ & $7.25 \pm 0.25$ & $\ldots$ & $\ldots$ & $1.80 \pm 0.18$ \\
\hline $7754 \ldots \ldots \ldots$ & $28.08 \pm 0.98$ & $7.40 \pm 0.26$ & $\ldots$ & $\ldots$ & $\ldots$ \\
\hline $7757 \ldots \ldots \ldots$ & $30.38 \pm 1.06$ & $6.75 \pm 0.24$ & $\ldots$ & $2.17 \pm 0.13$ & $2.05 \pm 0.20$ \\
\hline $7758 \ldots \ldots \ldots$ & $29.10 \pm 1.02$ & $6.95 \pm 0.24$ & $\ldots$ & $2.24 \pm 0.13$ & $\ldots$ \\
\hline $7759 \ldots \ldots \ldots$ & $\ldots$ & $6.42 \pm 0.22$ & $\ldots$ & $\ldots$ & $1.87 \pm 0.19$ \\
\hline $7765 \ldots \ldots \ldots$ & $\ldots$ & $6.42 \pm 0.22$ & $\ldots$ & $\ldots$ & $2.76 \pm 0.27$ \\
\hline $7766 \ldots \ldots \ldots$ & $27.38 \pm 0.96$ & $7.33 \pm 0.26$ & $3.04 \pm 0.18$ & $2.13 \pm 0.13$ & $2.54 \pm 0.25$ \\
\hline $7767 \ldots \ldots \ldots$ & $28.10 \pm 0.98$ & $6.95 \pm 0.24$ & $3.29 \pm 0.20$ & $2.29 \pm 0.13$ & $2.63 \pm 0.26$ \\
\hline $7769 \ldots \ldots \ldots$ & $29.80 \pm 1.04$ & $\ldots$ & $\ldots$ & $2.13 \pm 0.13$ & \\
\hline $7777 \ldots \ldots \ldots$ & $\ldots$ & $6.71 \pm 0.23$ & $3.39 \pm 0.20$ & $2.18 \pm 0.13$ & $2.66 \pm 0.26$ \\
\hline $7778 \ldots \ldots \ldots$ & $29.90 \pm 1.05$ & $7.50 \pm 0.26$ & $3.78 \pm 0.23$ & $2.12 \pm 0.13$ & $2.91 \pm 0.29$ \\
\hline $7779 \ldots \ldots \ldots$ & $29.22 \pm 1.02$ & $7.55 \pm 0.26$ & $3.38 \pm 0.20$ & $2.14 \pm 0.13$ & $2.70 \pm 0.27$ \\
\hline $7797 \ldots \ldots \ldots$ & $\ldots$ & $7.74 \pm 0.27$ & $3.68 \pm 0.22$ & $2.44 \pm 0.15$ & $2.70 \pm 0.27$ \\
\hline $7809 \ldots \ldots \ldots$ & $\ldots$ & $7.90 \pm 0.28$ & $\ldots$ & $2.44 \pm 0.15$ & $\ldots$ \\
\hline
\end{tabular}

${ }^{a}$ Fluxes in units of $10^{-13} \mathrm{ergs} \mathrm{s}^{-1} \mathrm{~cm}^{-2}$. 


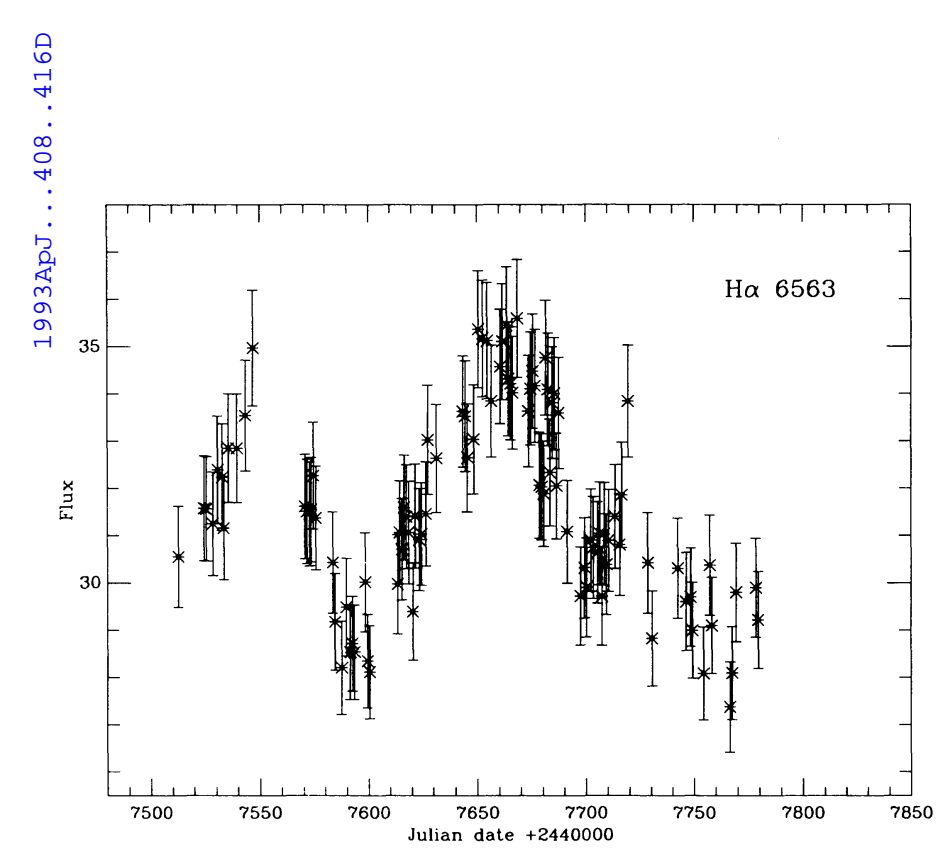

FIG. $5 a$

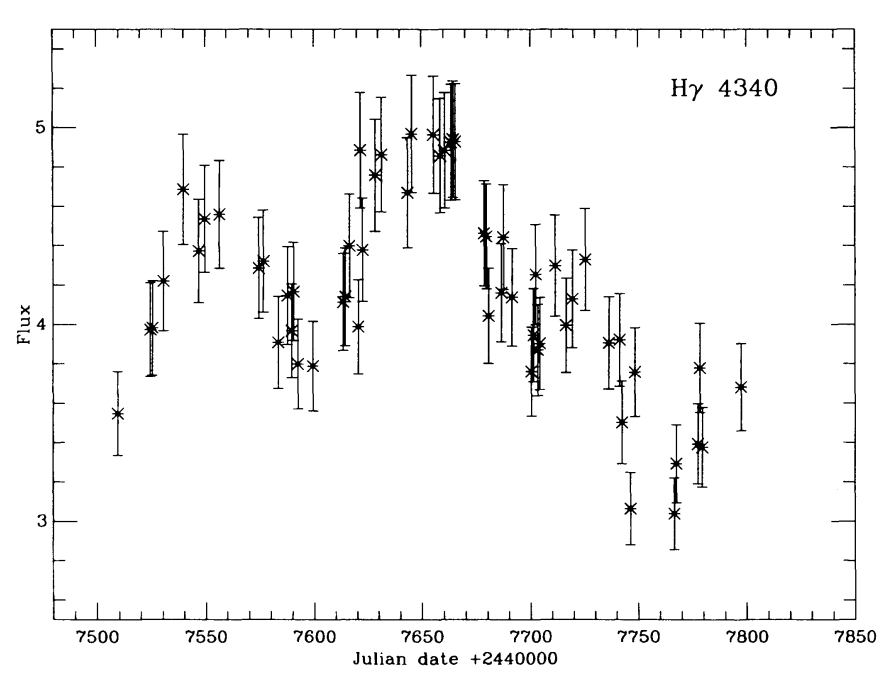

FIG. $5 c$

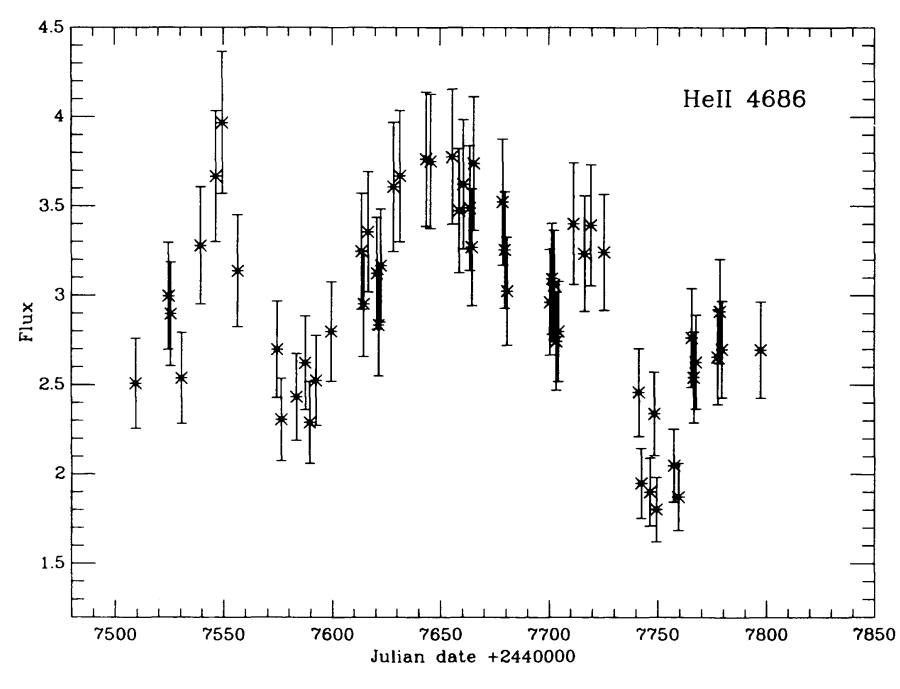

FIG. $5 e$

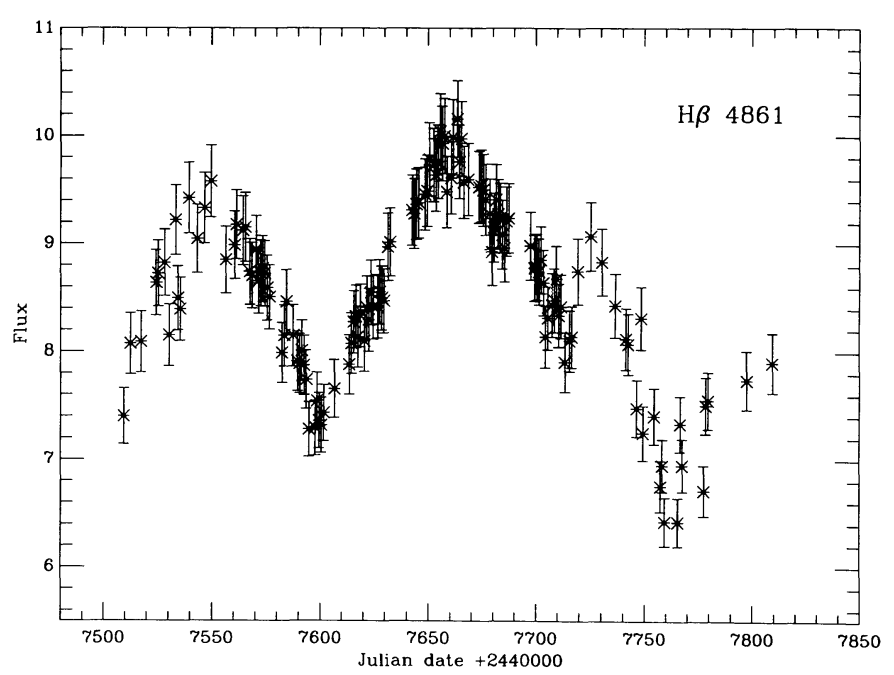

FIG. $5 b$

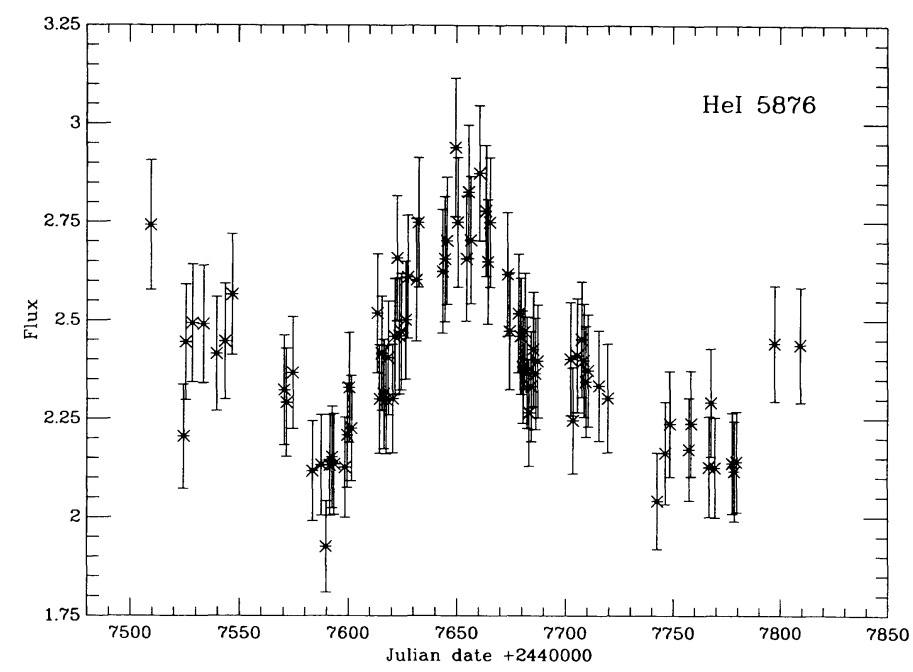

Fig. $5 d$

FIG. 5. - (a) Light curve for $\mathrm{H} \alpha$. The fluxes are in units of $10^{-13} \mathrm{ergs} \mathrm{s}^{-1}$ $\mathrm{cm}^{-2}$ and include the constant narrow-line component of $\mathrm{H} \alpha$ and the [N II] $\lambda \lambda 6548,6854$ lines (as observed through a $2^{\prime \prime} \times 10^{\prime \prime}$ aperture). (b) Light curve for $\mathrm{H} \beta$. The fluxes aere in units of $10^{-13} \mathrm{ergs} \mathrm{s}^{-1} \mathrm{~cm}^{-2}$ and include the constant narrow-line component of $\mathrm{H} \beta$ (as observed through a $2^{\prime \prime} \times 10^{\prime \prime}$ aperture). (c) Light curve for $\mathrm{H} \gamma$. The fluxes are in units of $10^{-13} \mathrm{ergs} \mathrm{s}^{-1} \mathrm{~cm}^{-2}$ and include the constant narrow-line component of $\mathrm{H} \gamma$ and the [O III] $\lambda 4363$ line (as observed through a 2" $\times 10^{\prime \prime}$ aperture). (d) Light curve for $\mathrm{He} \mathrm{I} \lambda 5876$ The fluxes are in units of $10^{-13} \mathrm{ergs} \mathrm{s}^{-1} \mathrm{~cm}^{-2}$ and include the constant narrow-line component of $\mathrm{He}_{\mathrm{I}} \lambda 5876$ and other weaker narrow features (as observed through a $2^{\prime \prime} \times 10^{\prime \prime}$ aperture). (e) Light curve for He II $\lambda 4686$. The fluxes are in units of $10^{-13} \mathrm{ergs} \mathrm{s}^{-1} \mathrm{~cm}^{-2}$ and include the constant narrow-line component of $\mathrm{He}$ II $\lambda 4686$ and other weaker narrow features (as observed through a $2 " \times 10^{\prime \prime}$ aperture), as well as an apparently constant, but undetermined, contribution from blended $\mathrm{Fe}$ II lines. 
TABLE 5

VARIABILITY PARAMETERS

\begin{tabular}{|c|c|c|c|}
\hline Feature & Mean Flux ${ }^{a}$ & $F_{\text {var }}$ & $R_{\max }$ \\
\hline $\begin{array}{l}\text { Continuum }(1350 \AA)^{\mathrm{b}} \ldots \ldots \ldots \ldots \ldots \\
\text { Continuum }(5100 \AA)^{\mathrm{c}} \ldots \ldots \ldots \ldots \ldots\end{array}$ & $\begin{array}{l}43.6 \pm 14.1 \\
9.95 \pm 1.30\end{array}$ & $\begin{array}{l}0.318 \\
0.126\end{array}$ & $\begin{array}{l}4.57 \\
2.36\end{array}$ \\
\hline 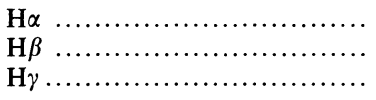 & $\begin{array}{r}31.6 \pm 2.0 \\
8.6 \pm 0.8 \\
4.2 \pm 0.5\end{array}$ & $\begin{array}{l}0.063 \\
0.096 \\
0.117\end{array}$ & $\begin{array}{l}1.30 \\
1.58 \\
1.6\end{array}$ \\
\hline $\begin{array}{l}\text { Не I } \lambda 5876 \ldots \ldots \ldots \ldots \ldots \ldots \ldots \ldots \\
\text { Не II } \lambda 46886 \ldots \ldots \ldots \ldots \ldots \ldots \ldots \ldots\end{array}$ & $\begin{array}{l}2.4 \pm 0.2 \\
3.0 \pm 0.5\end{array}$ & $\begin{array}{l}0.088 \\
0.182\end{array}$ & $\begin{array}{l}1.53 \\
2.20\end{array}$ \\
\hline
\end{tabular}

${ }^{\text {a }}$ Continuum fluxes in units of $10^{-15} \mathrm{ergs} \mathrm{s}^{-1} \mathrm{~cm}^{-2} \AA^{-1}$; line fluxes in units of $10^{-13} \mathrm{ergs} \mathrm{s}^{-1} \mathrm{~cm}^{-2}$.

b From data in Paper I (Clavel et al. 1991).

c From data in Paper III (Peterson et al. 1992).

\subsection{Time-Series Analysis of the Light Curves}

Simple time-series analysis which makes use of the emissionline and continuum cross-correlation functions (CCF) and line and continuum autocorrelation functions (ACF) can be used to infer basic characteristics of the BLR (e.g., Gaskell \& Sparke 1986; Gaskell \& Peterson 1987; Edelson \& Krolik 1988; Maoz \& Netzer 1989; Robinson \& Pérez 1990; Koratkar \& Gaskell 1991a, b). We have computed the ACFs and CCFs in two separate ways, by using the interpolation method (ICCF) as discussed by Gaskell \& Peterson (1987) and by using the discrete cross-correlation function (DCF) method proposed by Edelson \& Krolik (1988). The results of the ICCF and DCF methods are found to be consistent in the cases considered here.

The light curves of the optical emission lines given in Table 4 were cross-correlated with the UV continuum measurements at $1337 \AA$ from Paper I. The measurements we use here are those from the GEX-extracted IUE spectra (Reichert et al. 1991). The CCFs of the emission lines (computed by the ICCF method) are shown in Figures $6 a-6 e$. The CCFs of the emission lines all have well-defined peaks. The location of the peaks of the ICCF and DCF were determined by fitting a Gaussian curve to the correlation function starting at $50 \%$ of the maximum value. The confidence level of the CCF peaks, which was determined using the method described by Koratkar \& Gaskell (1991a), is very high for all of the emission lines, in each case higher than 0.996.

The results of the cross-correlation analysis are summarized in Table 7. Here $\tau_{\max }$ is the location of the CCF peak, and $r_{\max }$ is the value of the CCF at $\tau_{\max }$. As there is no generally accepted method of assessing the uncertainty in $\tau_{\max }$, we provide two separate estimates. The value $\sigma_{\tau(\mathbf{G P})}$ is determined by using equation (4) of Gaskell \& Peterson (1987). An alternative estimate, $\sigma_{\tau(\mathrm{MC})}$, can be obtained through Monte Carlo

TABLE 6

NARRow-Line CONTRIBUtions

\begin{tabular}{|c|c|}
\hline Feature & Flux $^{a}$ \\
\hline $\mathrm{H} \alpha$ complex & $2.910 \pm 0.280$ \\
\hline $\mathrm{H} \beta \ldots \ldots \ldots$ & $0.614 \pm 0.061$ \\
\hline $\mathbf{H} \gamma$ complex .. & $0.820 \pm 0.078$ \\
\hline He I $\lambda 5876 \ldots \ldots \ldots$ & $0.17 \pm 0.06$ \\
\hline Не II $\lambda 4686 \ldots \ldots \ldots \ldots \ldots$ & $0.17 \pm 0.06$ \\
\hline
\end{tabular}

simulations (e.g., Gaskell \& Peterson 1987; Maoz \& Netzer 1989). In our simulations, we adopted an interpolated and slightly smoothed version of the UV continuum as used in the cross-correlation analysis above. We then modeled the emission-line response by assuming a transfer function appropriate for a thin spherical shell (for simplicity, as only one parameter need to be specified) with a radius chosen to produce the appropriate emission-line lag for the particular line in question. We then sampled the model emission-line light curve in such a way as (a) to duplicate the number of actual data points that contribute to each light curve (e.g., 99 for $\mathrm{H} \alpha$, 57 for $\mathrm{He}$ II 24686 ) and (b) to preserve the same distribution of intervals between observations as in the original data. We then applied Gaussian-distributed random errors to each data point so as to obtain a value of $F_{\text {var }}$ identical to that of the real data for each emission line. The simulated continuum and emissionline light curves were then cross-correlated by the interpolation method, and the location of $\tau_{\max }$ was recorded for each trial. This procedure was repeated a larger number of times in order to build up a cross-correlation peak distribution (CCPD; Maoz \& Netzer 1989). In each case, the resulting CCPD was found to be quite symmetric about the mean expected from the model, so we quote only the rms width of the CCPD, which we than adopt as the error estimate $\sigma_{\tau(\mathrm{MC})}$.

Also given in Table 7 are the positions of the centers of the various CCFs at $0 \%, 30 \%$, and $50 \% r_{\text {max }}$.

The emission-line ACF can also provide important information about the BLR (see Gaskell \& Peterson 1987; Robinson \& Peréz 1990), and we therefore also computed the ACFs for each of the emission lines in this study. We note that the observed ACF as computed by the interpolation method $\mathrm{ACF}{ }_{\text {obs }}$ is the convolution of the true ACF with the ACF of the sampling window, $A C F_{\text {Sw }}$ (Gaskell \& Peterson 1987)

$$
\mathrm{ACF} \text { obs }=A C F_{\text {true }} * A C F_{\text {SW }} \text {. }
$$

The sampling window ACF is a measure of how much of the observed ACF is attributable to the apparent correlation that arises from interpolating between closely spaced data points. In order to assess how much the true ACF might be broadened by the sampling window, we calculated first $A C F_{\text {Sw }}$ for the optical data. The widths of $A C F_{\text {Sw }}$ are very small for all of the emission lines (Figs. $6 a-6 e$ ), ranging from 1.6 days for $\mathrm{H} \beta$ to 3.2

TABLE 7

Cross-Relation Results

\begin{tabular}{|c|c|c|c|c|c|c|c|c|}
\hline \multirow[b]{2}{*}{ LINE } & \multirow[b]{2}{*}{$\begin{array}{c}\tau_{\max } \\
\text { (days) }\end{array}$} & \multirow[b]{2}{*}{$\begin{array}{c}\sigma_{\tau(\mathrm{GP})} \\
\text { (days) }\end{array}$} & \multirow[b]{2}{*}{$\begin{array}{l}\sigma_{\tau(\mathrm{MC})} \\
\text { days) }\end{array}$} & \multirow[b]{2}{*}{$r_{\max }$} & \multirow{2}{*}{$\begin{array}{c}\text { FWHM } \\
\text { (days) }\end{array}$} & \multicolumn{3}{|c|}{ CENTER $^{a}$ (days) } \\
\hline & & & & & & 0.0 & 0.3 & 0.5 \\
\hline \multicolumn{9}{|c|}{ ICCF Method } \\
\hline $\mathrm{H} \alpha$. & 17.2 & 1.8 & 2.2 & 0.84 & 44.6 & 17.6 & 17.2 & 16.9 \\
\hline $\mathrm{H} \beta \ldots$ & 19.1 & 1.5 & 2.4 & 0.89 & 45.8 & 20.3 & 20.0 & 20.0 \\
\hline $\mathbf{H} \gamma \ldots \ldots \ldots \ldots$ & 12.5 & 2.3 & 2.1 & 0.89 & 47.1 & 13.2 & 12.9 & 12.6 \\
\hline He I $\lambda 5876 \ldots$. & 10.5 & 2.2 & 2.4 & 0.82 & 47.7 & 9.9 & 10.2 & 10.5 \\
\hline He II $\lambda 4686 \ldots \ldots$ & 7.1 & 2.1 & 1.4 & 0.85 & 40.6 & 7.3 & 6.1 & 7.0 \\
\hline \multicolumn{9}{|c|}{ DCF Method } \\
\hline $\mathrm{H} \alpha \ldots \ldots \ldots \ldots$ & 19.0 & 1.7 & & 0.75 & 41.6 & 19.4 & 20.5 & 19.9 \\
\hline $\mathbf{H} \beta \ldots \ldots \ldots \ldots$ & 21.9 & 1.0 & .. & 0.90 & 37.9 & 22.7 & 21.9 & 21.7 \\
\hline $\mathbf{H} \gamma \ldots \ldots \ldots \ldots$ & 12.9 & 2.5 & $\ldots$ & 0.73 & 44.3 & 13.7 & 13.2 & 13.9 \\
\hline He I $\lambda 5876 \ldots$. & 11.2 & 1.7 & $\ldots$ & 0.78 & 38.2 & 11.5 & 12.1 & 12.4 \\
\hline He II $\lambda 4686 \ldots$. & 9.8 & 1.5 & $\ldots$ & 0.91 & 30.9 & 11.3 & 10.6 & 10.0 \\
\hline
\end{tabular}

a Center of the CCF at various amplitudes, as fraction of $r_{\max }$. 

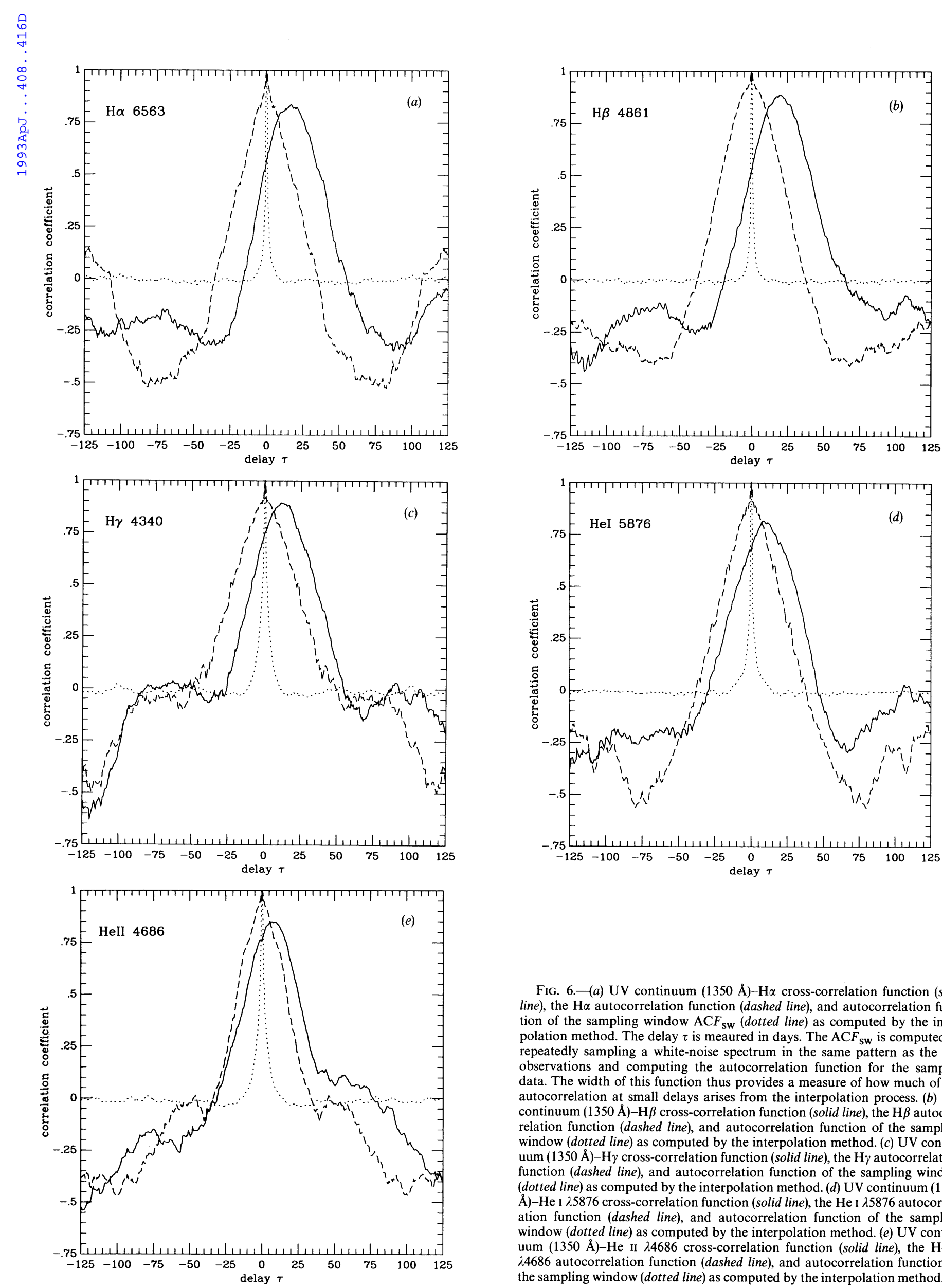

FIG. 6.-(a) UV continuum (1350 $\AA$ )-H $\alpha$ cross-correlation function (solid line), the $\mathrm{H} \alpha$ autocorrelation function (dashed line), and autocorrelation function of the sampling window $\mathrm{AC} F_{\mathrm{Sw}}$ (dotted line) as computed by the interpolation method. The delay $\tau$ is meaured in days. The $\mathrm{AC} F_{\mathrm{sw}}$ is computed by repeatedly sampling a white-noise spectrum in the same pattern as the real observations and computing the autocorrelation function for the sampled data. The width of this function thus provides a measure of how much of the autocorrelation at small delays arises from the interpolation process. (b) UV continuum (1350 $\AA)-\mathrm{H} \beta$ cross-correlation function (solid line), the $\mathrm{H} \beta$ autocorrelation function (dashed line), and autocorrelation function of the sampling window (dotted line) as computed by the interpolation method. (c) UV continuum (1350 $\AA$ ) $-\mathbf{H} \gamma$ cross-correlation function (solid line), the $\mathbf{H} \gamma$ autocorrelation function (dashed line), and autocorrelation function of the sampling window (dotted line) as computed by the interpolation method. (d) UV continuum (1350 $\AA$ ) - $\mathrm{He}$ I $\lambda 5876$ cross-correlation function (solid line), the $\mathrm{He}$ I $\lambda 5876$ autocorrelation function (dashed line), and autocorrelation function of the sampling window (dotted line) as computed by the interpolation method. (e) UV continuum $(1350 \AA)-\mathrm{He}$ II $\lambda 4686$ cross-correlation function (solid line), the He II $\lambda 4686$ autocorrelation function (dashed line), and autocorrelation function of the sampling window (dotted line) as computed by the interpolation method. 
TABLE 8

AutOCORRELATION Results

\begin{tabular}{cccccc}
\hline \hline Line & $\begin{array}{c}\text { FWHM } \\
\text { (ICCF) } \\
\text { (days) }\end{array}$ & $\begin{array}{c}\text { FWHM } \\
\text { (DCF) } \\
\text { (days) }\end{array}$ & $\begin{array}{c}\sigma_{\tau} \\
\text { (days) }\end{array}$ & $\begin{array}{c}\text { HWZI } \\
\text { (ICCF) } \\
\text { (days) }\end{array}$ & $\begin{array}{c}\text { HWZI } \\
\text { (DCF) } \\
\text { (days) }\end{array}$ \\
\hline $\mathrm{H} \alpha \ldots \ldots \ldots \ldots \ldots \ldots$ & 33.0 & 33.7 & 1.2 & 35.5 & 35.7 \\
$\mathrm{H} \beta \ldots \ldots \ldots \ldots \ldots \ldots$ & 41.2 & 43.1 & 1.3 & 33.8 & 37.4 \\
$\mathrm{H} \gamma \ldots \ldots \ldots \ldots \ldots \ldots$ & 43.0 & 44.2 & 1.9 & 39.0 & 40.8 \\
$\mathrm{He}$ i $\lambda 5876 \ldots \ldots \ldots$ & 43.3 & 37.9 & 1.4 & 34.1 & 38.1 \\
He II $\lambda 4686 \ldots \ldots \ldots$ & 38.2 & 34.2 & 1.5 & 27.2 & 33.4 \\
\hline
\end{tabular}

days for He II $\lambda 4686$. These values are much smaller than the widths of any of the emission-line ACFs or CCFs. This result justifies our assumption that the observed ACF is nearly identical to the true ACF and a correction for the limited sampling rate is not necessary.

The ACFs for the five emission-line light curves in this study are shown in Figures $6 a-6 e$. The basic characteristics of the observed ACFs are given in Table 8, as computed by both the DCF and ICCF methods; the full width at half-maximum (FWHM), half width at zero intensity (HWZI) and the error in the peak position, calculated from equation (4) of Gaskell \& Peterson (1987), are given.

\section{CONCLUSIONS}

The combined NGC 5548 ultraviolet and optical monitoring program has provided a data base of unprecedented size and quality for the study of emission-line variability in AGNs. In this contribution, we expand on previous analyses of these data and present light curves for all of the strong well-observed optical lines for the period 1988 December to 1989 October. Well-sampled light curves are presented for $\mathrm{H} \alpha$ ( 99 epochs), $\mathrm{H} \beta$ (133 epochs), $\mathrm{H} \gamma$ (58 epochs), He I $\lambda 5876$ (82 epochs), and He II $\lambda 4686$ (57 epochs). The optical $\mathrm{Fe}$ II multiplets were also examined for variability, but any real variations of these blends appear to be masked by relatively large systematic errors in measuring these features. The new $\mathrm{H} \beta$ light curve obtained here is in excellent agreement with the $\mathrm{H} \beta$ light curve of Paper II. These emission-line light curves all show qualitatively the same general pattern of variability that is seen in the UV continuum and lines and in the optical continuum. Crosscorrelation of the optical emission-line light curves with the UV continuum light curve reveals that the lines respond to the continuum variations with time delays ranging from around 7 days (for $\mathrm{He}$ II 24686 ) to around 20 days (for $\mathrm{H} \alpha$ and $\mathrm{H} \beta$ ). $\mathrm{H} \gamma$ responds more rapidly than the lower-order Balmer lines, and there is a general trend towards higher amplitude variations in higher-order lines. The time lags for the various lines are consistent with the general pattern of radial ionization stratification of the BLR deduced from the cross-correlation results for the UV emission lines (Paper I).

We are grateful the following individuals who contributed spectra which were used in this study: E. S. Barker, A. L. Cochran, and S. R. Sawyer (Texas), M. J. Bolte, D. Bond, D. Crampton, A. Ferguson, L. Saddlemyer, and P. F. Younger (DAO), R. D. Cohen (UCSD), J. P. Halpern (Columbia), D. Maoz (IAS), S. L. Morris and R. J. Weymann (OCIW), and T. J. Turner (GSFC). We wish to acknowledge support for this work through the following: BMFT grant Verbundforschung Astronomie FKZ 05 5GO42A1 (Universitäts-Sternwarte Göttingen); the National Science Foundation: AST-8915258 (Ohio State Univ.), AST-8714937 (Univ. Texas), RII-8800600 and AST-9058510 (Univ. Arizona), AST-8957063 and AST9003829 (Univ. California, Berkeley), AST-8611457 (Univ. of California, Santa Cruz), AST-8614510 (Univ. California), and NASA: NAG5-1366 (Ohio State Univ.), NAS5-29293 (Univ. Arizona), NAS8-30751 (Center for Astrophysics); the US-Israel Binational Science Foundation (grant 85/00085 to Wise Observatory); the National Science and Engineering Research Council of Canada (Mount Royal College). The Isaac Newton and William Herschel telescopes are run by the Royal Greenwich Observatory at the Spanish Observatorio del Roque de los Muchachos of the Instituto de Astrofísica de Canarias.
Antonucci, R. R. J., \& Cohen, R. D. 1983, ApJ, 271, 564 Clavel, J., et al. 1990, MNRAS, 246, 668

Clavel, J., Wamsteker, W., \& Glass, I. S. 1989, ApJ, 337, 236

Clavel, J., et al. 1991, ApJ, 366, 64 (Paper I)

Edelson, R. A., \& Krolik, J. H. 1988, ApJ, 333, 646

Gaskell, C. M., \& Peterson, B. M. 1987, ApJS, 65, 1

Gaskell, C. M., \& Sparke, L. S. 1986, ApJ, 305, 175

Koratkar, A. P., \& Gaskell, C. M. 1989, ApJ, 345, 637 .1991a, ApJ, 375, 85 1991b, ApJS, 75,719

Koratkar, A. P., \& MacAlpine, G. M. 1992, ApJ, 401, 11

Maoz, D., \& Netzer, H. 1989, MNRAS, 236, 21

Maoz, D., et al. 1990, ApJ, 351, 75

Maoz, D., et al. 1991, ApJ, 367, 493

Maoz, D., et al. 1993, ApJ, 404, 576

Netzer, H., et al. 1990, ApJ, 353, 108

Penston, M. V. 1991, in Variability of Active Galactic Nuclei, ed. H. R. Miller

\& P. J. Wiita (Cambridge: Cambridge Univ. Press), 343

\section{REFERENCES}

Peterson, B. M. 1987, ApJ, 312, 79

1988, PASP, 100, 18

Peterson, B. M., et al. 1991, ApJ, 368, 119 (Paper II) .1992, ApJ, 392, 470 (Paper III)

Peterson, B. M., \& Gaskell, C. M. 1991, ApJ, 368, 152

Peterson, B. M., Korista, K. T., \& Wagner, R. M. 1989, AJ, 98, 500

Peterson, B. M., Reichert, G. A., Korista, K. T., \& Wagner, R. M. 1990, ApJ, 352,68

Reichert, G., et al. 1991, in Variability of Active Galactic Nuclei, ed. H. R.

Miller \& P. J. Wiita (Cambridge: Cambridge Univ. Press), 335

Robinson, A., \& Pérez, E. 1990, MNRAS, 244, 138

Wamsteker, W., et al. 1990, ApJ, 354,446

Wilson, A. S., \& Ulvestad, J. S. 1982, ApJ, 260, 56

Wilson, A. S., Wu, X., Heckman, T. M., Bladwin, J. A., \& Balick, B. 1989, ApJ, 339,729

Winge, C., Pastoriza, M. G., Storchi-Bergmann, T., \& Lipari, S. 1992, ApJ, 393, 